Pure and Applied Mathematics Quarterly

Volume 9, Number 2

(Special Issue: In honor of

Dennis Sullivan, Part 2 of 2)

$291-332,2013$

\title{
Curvatures of Sobolev Metrics on Diffeomorphism Groups
}

\author{
B. Khesin, J. Lenells, G. Misiołek and S. C. Preston \\ To Dennis Sullivan on the occasion of his 70th birthday
}

\begin{abstract}
Many conservative partial differential equations correspond to geodesic equations on groups of diffeomorphisms. Stability of their solutions can be studied by examining sectional curvature of these groups: negative curvature in all sections implies exponential growth of perturbations and hence suggests instability, while positive curvature suggests stability. In the first part of the paper we survey what we currently know about the curvature-stability relation in this context and provide detailed calculations for several equations of continuum mechanics associated to Sobolev $H^{0}$ and $H^{1}$ energies. In the second part we prove that in most cases (with some notable exceptions) the sectional curvature assumes both signs.
\end{abstract}

Keywords: Riemannian metrics, diffeomorphism groups, sectional curvature, stability, Euler-Arnold equations.

\section{Contents}

1. Introduction 292

2. Preliminaries: metrics and geodesic equations 294

2.1. The Euler-Arnold equations on Lie groups: examples 295

Received September 6, 2011.

AMS Subject Classification(2000): 53C21, 58D05, 58D17. 
2.2. Further examples of infinite-dimensional geodesic equations

3. Global aspects of infinite-dimensional Riemannian geometry 302

3.1. Degenerate distance functions 302

3.2. Completeness and minimizing geodesics 303

3.3. Exponential map and extendability of geodesics 303

4. Jacobi fields, curvature, and stability 306

5. The sign of the curvature: previous results 312

6. The sign of the curvature: the one dimensional case 315

7. The sign of the curvature: higher dimensions 319

7.1. The $H^{1}$-metric on $\operatorname{Diff}\left(\mathbb{T}^{n}\right)$ : the EPDiff equation 320

7.2. The $L^{2}$-metric on $\operatorname{Diff}\left(\mathbb{T}^{n}\right)$ : the Burgers equation 322

7.3. The homogeneous $\dot{H}^{1}$-metric on $\operatorname{Diff}_{\mu, \text { ex }}\left(\mathbb{T}^{2}\right) \quad 323$

Appendix A. Semi-direct products and curvature formulas 325

$\begin{array}{ll}\text { References } & 328\end{array}$

\section{Introduction}

The idea that stability of a dynamical system can be investigated using tools of Riemannian geometry goes back to Hadamard [22], who studied the free motion of a particle on a surface of constant negative curvature. A similar approach was considered by Synge [68]. Perhaps the most influential example is due to Arnold [1], who showed that fluid motions can be viewed as geodesics in the infinitedimensional group of volume-preserving diffeomorphisms (volumorphisms). This led him to examine curvature of the volumorphism group and derive a number of results on stability of ideal fluids. Roughly speaking, on a finite-dimensional manifold negative sectional curvature is related to instability, and positive sectional curvature is related to stability of the corresponding geodesic flow; the Rauch comparison theorem makes this comparison rigorous. Since the work of Arnold, other partial differential equations have been interpreted as geodesic equations 
in infinite-dimensional spaces and, as with ideal fluids, calculating sectional curvatures in these cases has become a matter of broader interest.

In this paper we examine the sign of the sectional curvature of certain metrics on infinite-dimensional manifolds (which are associated with several well-known equations of mathematical physics) and its relevance in the stability analysis of the associated initial value problems.

Our main interest is in those equations that arise from right-invariant metrics on the group of (smooth) diffeomorphisms Diff $(M)$, or its subgroup of volumepreserving diffeomorphisms $\operatorname{Diff}_{\mu}(M)$, of a compact $n$-dimensional manifold $M$ without boundary. Both spaces can be completed to Hilbert manifolds Diffs ${ }^{s}(M)$ and $\operatorname{Diff}_{\mu}^{s}(M)$ modeled on Sobolev spaces of $H^{s}$ vector fields and divergence-free vector fields, respectively, with $s>n / 2+1$. We assume that $M$ has a Riemannian metric $\langle\cdot, \cdot\rangle$ with volume form $\mu$. We equip the groups with right-invariant Sobolev metrics such that, on the tangent space at a diffeomorphism $\eta$, we have

$$
\langle\langle u \circ \eta, v \circ \eta\rangle\rangle_{\eta}=\int_{M}\left(a\langle u, v\rangle+b\left\langle\delta u^{b}, \delta u^{b}\right\rangle+c\left\langle d u^{b}, d u^{b}\right\rangle\right) d \mu,
$$

for any vector fields $u, v$ on $M$. Here $d$ is the exterior derivative, $\delta= \pm * d *$ is its (formal) adjoint, $a, b, c$ are non-negative constants, and $b$ and $\sharp$ denote the standard "musical isomorphisms" of the metric corresponding to lowering and raising of indices. Formula (1.1) simplifies in dimensions $n \leq 3$ where we have ${ }^{1}$

$$
\langle\langle u \circ \eta, v \circ \eta\rangle\rangle_{\eta}= \begin{cases}\int_{M}\left(a u v+b u_{x} v_{x}\right) d x & n=1, \\ \int_{M}(a\langle u, v\rangle+b \operatorname{div} u \cdot \operatorname{div} v+c \operatorname{curl} u \cdot \operatorname{curl} v) d A & n=2, \\ \int_{M}(a\langle u, v\rangle+b \operatorname{div} u \cdot \operatorname{div} v+c\langle\operatorname{curl} u, \operatorname{curl} v\rangle) d V & n=3 .\end{cases}
$$

Using the metric (1.1), one derives a number of PDE that are of interest in continuum mechanics and geometry. For example, in the one-dimensional case one obtains Burgers' equation, the Camassa-Holm equation, and the HunterSaxton equation as geodesic equations for appropriate choices of $a$ and $b$. In higher dimensions one gets the EPDiff equation and the so-called template-matching equation. Furthermore, projection onto $\operatorname{Diff}_{\mu}(M)$ yields the usual Euler equations

\footnotetext{
${ }^{1}$ Recall that $\operatorname{curl} u=* d u^{b}$ and $\operatorname{sgrad} f=-(\delta * f)^{\sharp}$ in two dimensions, while curl $u=\left(* d u^{b}\right)^{\sharp}$ in three dimensions.
} 
of hydrodynamics and the Lagrangian-averaged Euler- $\alpha$ equation. We refer to the paper [31] and the references therein for more details.

The goal of this paper is two-fold. In the first part we describe some aspects of Riemannian geometry of infinite-dimensional manifolds which are relevant to the analysis of partial differential equations of mathematical physics. In particular, we review the framework of the Euler-Arnold equations on Lie groups equipped with right-invariant metrics and explain the role played by sectional curvature in the study of (Lagrangian) stability.

In the second part we present new results on the sign of the sectional curvature for the metric (1.1) on $\operatorname{Diff}(M)$ and $\operatorname{Diff}_{\mu}(M)$ for different choices of the parameters $a, b$ and $c$. In order to simplify calculations, we will take $M$ to be either the circle $S^{1}$ or the flat torus $\mathbb{T}^{n}$. We show that in most cases the sectional curvature assumes both signs. Two notable exceptions are: $b=c=0$ and $n=1$, in which case the sectional curvature of $\operatorname{Diff}\left(S^{1}\right)$ turns out to be non-negative; and $a=c=0$ and $n \geq 1$, in which case the sectional curvature of the quotient space $\operatorname{Diff}(M) / \operatorname{Diff}_{\mu}(M)$ is strictly positive for any compact $M$. The latter case is studied in detail in the paper [31]. For the $H^{1}$ metric on $\operatorname{Diff}\left(S^{1}\right)$ we obtain a simple curvature expression and explain how it can be viewed as the Gauss-Codazzi formula for an isometric embedding of the group of circle diffeomorphisms in a larger space.

Besides the fact that right-invariant $H^{1}$ metrics (1.1) arise as Lagrangians of many PDE of continuum mechanics, our motivation to study them is also purely geometric: they are the natural metrics induced on orbits of pullback actions on spaces of tensor fields. For example, the canonical $L^{2}$ metric on the space of all Riemannian metrics on a compact manifold $M$ induces a metric of the type (1.1) on $\operatorname{Diff}(M)$ (viewed as an orbit of any particular metric under the pullback action) whose geometry was studied in [31].

\section{Preliminaries: metrics and geodesic equations}

In this section we review some well-known examples of infinite-dimensional Riemannian manifolds and their geodesic equations. Most interesting from our point of view are infinite-dimensional Lie groups, especially diffeomorphism groups equipped with right-invariant metrics. We describe examples of such groups 
in Section 2.1. In Section 2.2 we discuss other situations of interest when the manifolds are not Lie groups or the metrics are not right-invariant.

2.1. The Euler-Arnold equations on Lie groups: examples. We begin by describing the general Lie-theoretic setup of Arnold [1].

Consider an infinite-dimensional Lie group $G$ equipped with a smooth rightinvariant (weak) Riemannian metric determined by an inner product $\langle\langle\cdot, \cdot\rangle\rangle$ defined on the tangent space $T_{e} G$ at the identity element. A geodesic in the group starting from $e$ in the direction $u_{0}$ can be obtained from the solution $u(t)$ of the Cauchy problem for the associated Euler-Arnold equation on $T_{e} G$, namely

$$
\frac{d u}{d t}=-\operatorname{ad}_{u}^{*} u, \quad u(0)=u_{0}
$$

where for any fixed $v \in T_{e} G$, the operator $u \rightarrow \operatorname{ad}_{v}^{*} u$ is the adjoint of the linear operator $u \rightarrow \operatorname{ad}_{v} u=-[v, u]$ with respect to the inner product on $T_{e} G$; specifically, ${ }^{2}$

$$
\left\langle\left\langle\operatorname{ad}_{v}^{*} u, w\right\rangle\right\rangle=-\langle\langle u,[v, w]\rangle\rangle, \quad u, v, w \in T_{e} G .
$$

The geodesic is then obtained by solving the flow equation

$$
\frac{d \eta}{d t}=D R_{\eta(t)} u(t), \quad \eta(0)=e
$$

where $\xi \mapsto R_{\eta}(\xi)$ denotes the right-translation in $G$ by $\eta$.

In the case of ideal hydrodynamics, $G$ is the group of volumorphisms of a manifold $M$,

$$
G=\operatorname{Diff}_{\mu}(M):=\left\{\eta \in \operatorname{Diff}(M) \mid \eta^{*} \mu=\mu\right\},
$$

with the right-invariant metric given at the identity by the $L^{2}$ inner product, i.e., by setting $b=c=0$ in (1.1). The resulting Euler-Arnold equations (2.1) are the familiar Euler equations of incompressible fluids in $M$

$$
u_{t}+\nabla_{u} u=-\nabla p, \quad \operatorname{div} u=0,
$$

sometimes written in the form

$$
\omega_{t}+\mathcal{L}_{u} \omega=0
$$

\footnotetext{
${ }^{2}$ Here $[\cdot, \cdot]$ denotes the commutator on $T_{e} G$ induced by the Lie bracket of right-invariant vector fields on $G$, i.e. $[u, v]=[X, Y]_{e}$ where $X, Y$ are the right-invariant vector fields determined by $X_{e}=u$ and $Y_{e}=v$.
} 
where $\omega=\operatorname{curl} u$ is the vorticity. ${ }^{3}$

If we set $a=1$ and $c=\alpha^{2}$ with $b=0$ in (1.1) on the volumorphism group, then the corresponding Euler-Arnold equation (2.1) is called the Lagrangian-averaged Euler equation [24,64]. It is more complicated than (2.4), but its "vorticity" given by $\omega=\operatorname{curl} u-\alpha^{2} \Delta \operatorname{curl} u$ satisfies the same equation as (2.5). The analysis of this equation presents similar difficulties as (2.4) or (2.5); see e.g., [26].

Remark 2.1. The volumorphism group is one of the three "classical" diffeomorphism groups. Another is the symplectomorphism group $\operatorname{Diff}_{\omega}(M)$, consisting of diffeomorphisms preserving a symplectic form $\omega$ of an even-dimensional manifold $M$, and the third is the contactomorphism group $\operatorname{Diff}_{\alpha}(M)$ consisting of diffeomorphisms $\eta$ such that $\eta^{*} \alpha=F \alpha$, where $\alpha$ is a contact form and $F$ is a nowhere-zero function on an odd-dimensional manifold $M$. Geodesic equations of the right-invariant $L^{2}$ metric on these groups have been studied in [13, 29, 15]. In two dimensions the geodesic equation on the symplectomorphism group reduces to the Euler equation (2.4), and in one dimension the geodesic equation for contactomorphisms reduces to the Camassa-Holm equation. Simpler equations arise when the $L^{2}$ metric is restricted to the subgroup of Hamiltonian diffeomorphisms or the subgroup of strict contactomorphisms; see Smolentsev [67] for a review of their properties.

It is worth pointing out that the subgroup of Hamiltonian diffeomorphisms carries a bi-invariant metric given at the identity by

$$
\langle\langle\operatorname{sgrad} f, \operatorname{sgrad} g\rangle\rangle=\int_{M} f g d \mu,
$$

where $f$ and $g$ are assumed to have mean zero. For such metrics we have $\left\langle\left\langle u, \operatorname{ad}_{v} w\right\rangle\right\rangle+\left\langle\left\langle\operatorname{ad}_{v} u, w\right\rangle\right\rangle=0$ whenever $u, v$ and $w$ are in the Lie algebra. It follows that $\operatorname{ad}_{u}^{*} u=0$, and hence the Euler equation (2.1) reduces to $d u / d t=0$. Geodesics are easy to find as they are one-parameter subgroups: simply fix a velocity field and compute the flow.

The group of circle diffeomorphisms $\operatorname{Diff}\left(S^{1}\right)$ has been a rich source of examples. In this case we can set $c=0$ in the formula (1.1) since in one dimension $d u^{b}=0$ for any vector field $u$. Two much-studied Euler-Arnold equations that

${ }^{3}$ In two dimensions $\omega=\operatorname{curl} u$ is a function and $\mathcal{L}_{u} \omega=\langle u, \nabla \omega\rangle$; in three dimensions $\omega=\operatorname{curl} u$ is a vector field and $\mathcal{L}_{u} \omega=[u, \omega]$. 
arise here are the periodic (inviscid) Burgers equation

$$
u_{t}+3 u u_{x}=0
$$

associated with the $L^{2}$ inner product (with $b=0$ ) and the periodic Camassa-Holm equation

$$
u_{t}-u_{t x x}+3 u u_{x}-2 u_{x} u_{x x}-u u_{x x x}=0
$$

obtained from the $H^{1}$ product (with $a=b=1$ ); see [3, 51].

Interesting examples also arise on the Bott-Virasoro group $\operatorname{Vir}\left(S^{1}\right)$, the universal central extension of $\operatorname{Diff}\left(S^{1}\right)$, with a group law defined by

$$
(\eta, \alpha) \circ(\xi, \beta)=\left(\eta \circ \xi, \alpha+\beta+\frac{1}{2} \int_{S^{1}} \log \partial_{x}(\eta \circ \xi) d \log \partial_{x} \xi\right)
$$

where $\eta, \xi \in \operatorname{Diff}\left(S^{1}\right)$ and $\alpha, \beta \in \mathbb{R}$. The right-invariant metric on the BottVirasoro group given at the identity by the $L^{2}$ inner product

$$
\langle\langle(u, a),(v, b)\rangle\rangle_{L^{2}}=\int_{S^{1}} u v d x+a b,
$$

yields as its Euler-Arnold equation (2.1) the Korteweg-de Vries equation [3, 55]

$$
u_{t}+3 u u_{x}+a u_{x x x}=0, \quad a=\text { const. }
$$

If we use the $H^{1}$ inner product instead

$$
\langle\langle(u, a),(v, b)\rangle\rangle_{H^{1}}=\int_{S^{1}}\left(u v+u_{x} v_{x}\right) d x+a b,
$$

then the corresponding Euler-Arnold equation is the Camassa-Holm equation with drift [51]

$$
u_{t}-u_{t x x}+3 u u_{x}-2 u_{x} u_{x x}+(\kappa-u) u_{x x x}=0, \quad \kappa=\text { const. }
$$

Equations (2.7) and (2.8) have higher-dimensional analogues. For example, the $n$-dimensional version of the Burgers equation (2.7) arising from a right-invariant $L^{2}$ metric on $\operatorname{Diff}(M)$ is the so-called template-matching equation [54]

$$
u_{t}+\nabla_{u} u+(\nabla u)^{\dagger}(u)+(\operatorname{div} u) u=0
$$

while the $n$-dimensional version of the Camassa-Holm equation (2.8) on $\operatorname{Diff}(M)$ is the EPDiff equation [24].

Other examples of geodesic equations for right-invariant metrics on infinitedimensional groups include 
- the equation $\phi_{t}+u(\phi)=0$ of passive scalar motion coupled with (2.4) (on the semidirect product of $\operatorname{Diff}_{\mu}(M)$ with $C^{\infty}(M)$ );

- the equation of $3 D$ magnetohydrodynamics (on the semi-direct product of $\operatorname{Diff}_{\mu}(M)$ with divergence-free vector fields)

$u_{t}+\nabla_{u} u=-\nabla p+\nabla_{B} B, \quad B_{t}+[u, B]=0, \quad \operatorname{div} u=\operatorname{div} B=0 ;$

- the $\mu$-CH equation, corresponding to an $H^{1}$-type metric on $\operatorname{Diff}\left(S^{1}\right)$, see Remark 6.5 and [30];

- the quasigeostrophic equation in $\beta$-plane approximation on $\mathbb{T}^{2}$ on the central extension of the group of Hamiltonian diffeomorphisms

$$
\omega_{t}+\{\psi, \omega\}=-\beta \partial_{x} \psi, \quad \omega=\Delta \psi-\mathcal{F} \psi
$$

for some constants $\beta$ and $\mathcal{F}$ (see $[25]$ );

as well as the Boussinesq approximation to stratified fluids, equations for charged fluids and fluids in Yang-Mills fields, the Landau-Lifschitz equations as well as various 2-component generalizations of the one-dimensional equations mentioned above; see e.g., $[34,70]$ for details and more references.

\subsection{Further examples of infinite-dimensional geodesic equations.}

2.2.1. Spaces of curves. Let $\Omega M$ be the (free) loop space over a compact Riemannian manifold $M$ whose points are smooth maps from $S^{1}$ to $M .{ }^{4}$ The tangent space to $\Omega M$ at a point $\gamma$ consists of vector fields in $M$ along $\gamma$, i.e., maps $s \rightarrow V(s) \in T_{\gamma(s)} M$.

Two metrics on $\Omega M$ have been of particular interest. The first is the weak Riemannian $L^{2}$ metric given at $\gamma \in \Omega M$ by

$$
\langle\langle U, V\rangle\rangle_{L^{2}, \gamma}=\int_{S^{1}}\langle U(s), V(s)\rangle_{\gamma(s)} d s .
$$

Its geodesics correspond to geodesics on the underlying manifold: if $\eta(0)=\gamma$ and $\dot{\eta}(0)=V$, then $\eta(t)(s)=\exp _{\gamma(s)}(t V(s))$.

The other is the Sobolev $H^{1}$ metric

$$
\langle\langle U, V\rangle\rangle_{H^{1}, \gamma}=\int_{S^{1}}\left(\langle U(s), V(s)\rangle_{\gamma(s)}+\left\langle\frac{D U}{d s}, \frac{D V}{d s}\right\rangle_{\gamma(s)}\right) d s,
$$

\footnotetext{
${ }^{4}$ If $M$ is a Lie group then $\Omega M$ becomes a loop group under pointwise multiplication.
} 
where $D / d s$ denotes the covariant derivative along $\gamma$ in $M$. The metric (2.12) is in fact the more natural of the two because it turns the set $\overline{\Omega M}^{H^{1}}$ consisting of all $H^{1}$ loops in $M$ into a complete Hilbert Riemannian manifold.

The set of simple closed curves in $\mathbb{R}^{2}$ can also be regarded as a version of the loop space. Such curves may be viewed as boundaries of planar "shapes," and finding a suitable notion of distance between shapes has been of interest in applications to pattern theory. For this purpose, however, parameterizations are irrelevant; thus it is useful to pass to the quotient by the diffeomorphism group of $S^{1}$. Geodesics on the quotient of the metrics induced by (2.11) and (2.12) were studied by Michor and Mumford [46]. Another approach is to consider the subspace consisting of those curves parameterized by arc length (or its multiple) with the induced $L^{2}$ or $H^{1}$ metric. The $L^{2}$ geodesics on this subspace are solutions of a wave-like equation

$$
\eta_{t t}=\partial_{s}\left(\sigma \eta_{s}\right), \quad \sigma_{s s}-\left|\eta_{s s}\right|^{2} \sigma=-\left|\eta_{s t}\right|^{2}, \quad\left|\eta_{s}\right| \equiv 1,
$$

which describes an inextensible string (the asymptotic limit of a string with a very strong tension); see [61] for a geometric discussion of this equation.

2.2.2. Homogeneous spaces. Degenerate metrics on diffeomorphism groups also lead to natural geometries on their quotient spaces. For example, if $u$ is a vector field on $S^{1}$ and $\eta \in \operatorname{Diff}\left(S^{1}\right)$, then setting

$$
\langle\langle u \circ \eta, v \circ \eta\rangle\rangle_{\dot{H}^{1}}=\int_{S^{1}} u_{x} v_{x} d x
$$

one obtains an invariant degenerate Sobolev $\dot{H}^{1}$ metric which is a limiting case of the $H^{1}$ metric (1.2) when $a \rightarrow 0$ or $b \rightarrow \infty$. It becomes a weak Riemannian metric on the homogeneous space $\operatorname{Diff}\left(S^{1}\right) / S^{1}$. The corresponding Euler-Arnold equation is the Hunter-Saxton equation [32]

$$
u_{t x x}+2 u_{x} u_{x x}+u u_{x x x}=0 .
$$

Passing to the quotient space is geometrically appealing since the manifold turns out to be isometric to a subset of the round Hilbert sphere [36].

More generally, the same construction applies on any Riemannian manifold $M$ using the right-invariant degenerate metric

$$
\left\langle\langle u \circ \eta, v \circ \eta\rangle{\dot{H}^{1}}=\int_{M} \operatorname{div} u \cdot \operatorname{div} v d \mu,\right.
$$


on the quotient space $\operatorname{Diff}(M) / \operatorname{Diff}_{\mu}(M)$. Its Euler-Arnold equation is a higherdimensional analogue of (2.14) given by

$$
\nabla \operatorname{div} u_{t}+(\operatorname{div} u) \nabla \operatorname{div} u+\nabla\langle u, \nabla \operatorname{div} u\rangle=0,
$$

and one can establish a similar isometry with a subset of the round sphere in a Hilbert space. The induced Riemannian distance turns out to be a spherical analogue of the Hellinger metric in probability theory; see [31] for details.

2.2.3. Spaces of maps and non-invariant metrics. More generally, given a Riemannian manifold $M$ and a compact manifold $N$ with a volume form $\nu$ (and possibly with boundary), consider the space $C^{\infty}(N, M)$ of smooth maps from $N$ into $M$. On each tangent space at $f \in C^{\infty}(N, M)$, we can define an $L^{2}$ metric by

$$
\langle\langle U, V\rangle\rangle_{f}=\int_{M}\langle U(x), V(x)\rangle_{f(x)} d \nu(x),
$$

and, as in the case of the loop space and (2.11), show that its geodesics come directly from geodesics on $M$.

The group of smooth diffeomorphisms $\operatorname{Diff}(M)$ is an open subset of the Fréchet manifold $C^{\infty}(M, M)$, so that we can likewise put the metric (2.16) on it. Note that this metric is not right-invariant; nevertheless the corresponding geodesic equation can be rewritten on the tangent space to the identity, where it becomes the multidimensional inviscid Burgers (or pressureless compressible Euler) equation

$$
\frac{\partial u}{\partial t}+\nabla_{u} u=0
$$

Since families of geodesics in $M$ starting from nearby points will typically contain crossing geodesics (at which time the geodesic in $\operatorname{Diff}(M)$ must exit the diffeomorphism group), solutions of the pressureless Euler equation solutions in general will blow up in finite time. Physically, this corresponds to the emergence of a shock wave leading to collisions of the fluid (or gas) particles. Nonetheless, the geodesic remains in $C^{\infty}(M, M)$ for all time.

Remark 2.2. The equations of incompressible fluids with boundary can be viewed formally as geodesic equations on the space $\mathcal{E}_{\mu}\left(\Omega, \mathbb{R}^{n}\right)$ of volume-preserving embeddings of the closure $\bar{\Omega}$ of an open subset of $\mathbb{R}^{n}$ into $\mathbb{R}^{n}$. These equations were studied geometrically by Ebin [12] and shown to be identical to the standard 
equations of incompressible fluid mechanics except for the fact that the boundary condition for the pressure is a Dirichlet rather than a Neumann condition. However the free-boundary equations are not well-posed unless one adds an extra condition such as in [39].

2.2.4. Spaces of metrics. Another geometrically interesting space is the space $\operatorname{Met}(M)$ of all Riemannian metrics on a compact manifold $M$. For any metric $g$ on $M$ and any $\eta \in \operatorname{Diff}(M)$ we define the pullback metric $\eta^{*} g$. In [11], Ebin studied a Riemannian metric on $\operatorname{Met}(M)$ which is invariant under the pullback action. Given $g \in \operatorname{Met}(M)$ and tangent vectors $A$ and $B$ (smooth tensor fields of symmetric bilinear forms) the metric is defined by

$$
\langle\langle A, B\rangle\rangle_{g}=\int_{M} \operatorname{Tr}_{g}(A B) d \mu_{g}
$$

where $\operatorname{Tr}_{g}$ is the trace with respect to $g$ and $\mu_{g}$ is the Riemannian volume form. The curvature and geodesics of this metric were computed explicitly by Freed and Groisser [19]: sectional curvature is non-positive and geodesics generally exist only for finite time (until the metric becomes degenerate). The diffeomorphism group embeds in $\operatorname{Met}(M)$ as an orbit of a generic $g$ (i.e., with no non-trivial isometries), and if $g$ is Einstein then the induced metric on $\operatorname{Diff}(M)$ is a special case of (1.1). We refer to Clarke [8] for recent results on the distance and diameter of this space.

Similarly, one can endow the space of all volume forms $\operatorname{Vol}(M)$ on $M$ with a natural invariant metric defined for $n$-form fields $\alpha$ and $\beta$ tangent to $\mu$ by

$$
\langle\langle\alpha, \beta\rangle\rangle_{\mu}=\int_{M} \frac{\alpha}{\mu} \frac{\beta}{\mu} d \mu .
$$

Although this metric is flat, it is not geodesically complete in general. Orbits of the diffeomorphism group in $\operatorname{Vol}(M)$ are the homogeneous spaces of densities $\operatorname{Diff}(M) / \operatorname{Diff}_{\mu}(M)$ of constant positive curvature; we refer to [31].

The map $g \mapsto \mu_{g}$, where $\mu_{g}$ is the Riemannian volume of $g$, is a submersion and it becomes a Riemannian submersion after suitable rescaling of the metric on $\operatorname{Vol}(M)$. Its fibers are the spaces of metrics $\operatorname{Met}_{\mu}(M)$ with the same volume form $\mu$. These fibers are globally symmetric (with non-positive curvature and indefinitely extendable geodesics) in the induced metric from $\operatorname{Met}(M)$; see [11]. The natural action on $\operatorname{Met}_{\mu}(M)$ is pullback by volumorphisms, and if $g$ has no 
nontrivial isometries, then the orbits of $\operatorname{Diff}_{\mu}(M)$ are embedded submanifolds with right-invariant metrics.

\section{Global aspects of infinite-Dimensional Riemannian geometry}

The obstacles that arise in the study of global Riemannian geometry of infinitedimensional manifolds are well known. They are mostly caused by the lack of local compactness or the fact that the topology generated by the metric may be weaker than the manifold topology. As a result some of the finite-dimensional techniques are not available or are of limited use. For example, the Riemannian exponential map may not be defined on the whole tangent bundle or even be smooth, conjugate points may cluster along finite geodesic segments or have infinite multiplicity, etc. In this section we illustrate some of these situations with a few familiar examples.

3.1. Degenerate distance functions. The distance between two points in a weak Riemannian Hilbert manifold can be defined as in finite dimensions, i.e., as the infimum of lengths of piecewise smooth curves joining them. It is easy to prove that it satisfies all the axioms of a metric space except for nondegeneracy, which typically requires some additional assumptions.

As an example, consider the right-invariant $L^{2}$ metric on $\operatorname{Diff}(M)$ defined by

$$
\langle\langle u \circ \eta, v \circ \eta\rangle\rangle_{\eta}=\int_{M}\langle u, v\rangle d \mu,
$$

which corresponds to the case $b=c=0$ in (1.1). In [45] it is shown that the geodesic distance is identically zero (i.e., between any two diffeomorphisms there are curves of arbitrarily short length). This is essentially related to the lack of control over the Jacobian. The same phenomenon also occurs for the rightinvariant $L^{2}$ metric on the Bott-Virasoro group [5], for the $L^{2}$ metric on the "shape space" of curves modulo reparameterizations [46], and for the bi-invariant Hofer-type $L^{2}$ metric (2.6) on the Hamiltonian diffeomorphisms [17].

On the other hand, we obtain nondegenerate Riemannian distances for the $L^{2}$ metric on $\operatorname{Diff}_{\mu}(M)$ [14], the right-invariant metric on $\operatorname{Diff}(M)$ corresponding to $c=0$ in (1.1) [53], the space $C^{\infty}(N, M)$ of maps in the $L^{2}$ metric (2.16), the space of arc-length parameterized curves [61], and the space $\operatorname{Diff}(M) / \operatorname{Diff}_{\mu}(M)$ of densities [31]. 
3.2. Completeness and minimizing geodesics. Even if the geodesic distance is nondegenerate, thus providing a genuine metric space structure on a space of maps, this metric space may not be complete. For example, the completion of $C^{\infty}(N, M)$ in the non-invariant $L^{2}$ metric (2.16) consists of measurable maps from $N$ to $M$ which may not even be continuous. The same phenomenon occurs for the group of volumorphisms $\operatorname{Diff}_{\mu}(M)$ with the $L^{2}$ metric if $M$ is a threedimensional manifold - in this case the completion in the Riemannian distance is the space of all measure-preserving maps; see [65]. ${ }^{5}$

In finite dimensions, completeness of a Riemannian manifold $M$ as a metric space is equivalent to geodesic completeness, i.e., extendability of geodesics for all time, which in turn implies that any two points in $M$ can be joined by a minimal geodesic. The proof of this result, the Hopf-Rinow theorem, relies crucially on local compactness, and the result is no longer true in infinite dimensions as observed by Grossman [21] and Atkin [4]. Grossman constructed an infinite-dimensional ellipsoid in the space $\ell^{2}$ of square-summable sequences with points which cannot be connected by a minimal geodesic in the induced metric from $\ell^{2}$, and Atkin modified this construction to get points that cannot be joined by any geodesic at all. Interestingly, Ekeland [16] showed that on a complete Riemannian Hilbert manifold the set of points attainable from a given one with a minimizing geodesic contains a dense $G_{\delta}$ set (recall that a $G_{\delta}$ is a countable intersection of open sets).

One situation in which everything works nicely is the $H^{1}$ completion of the space of smooth loops $\overline{\Omega M^{H}}$. Unlike many other examples discussed here, it is a genuine (strong) Riemannian Hilbert manifold in the topology generated by the distance function of (2.12), and by the result of Eliasson [18] any two of its points can be joined by a minimizing geodesic.

3.3. Exponential map and extendability of geodesics. If the Cauchy problem for the geodesic equation on a (possibly weak) Riemannian Hilbert manifold $\mathcal{M}$ is locally well-posed, then the exponential map of $\mathcal{M}$ can be defined as in finite dimensions. Using the scaling properties of geodesics we set

$$
\exp _{p}: U \subset T_{p} \mathcal{M} \rightarrow \mathcal{M}, \quad \exp _{p}(v)=\gamma(1),
$$

where $\gamma(t)$ is the unique geodesic from $\gamma(0)=p$ with initial velocity $\dot{\gamma}(0)=v$ in some open neighborhood $U$ of zero in the tangent space at $p$.

${ }^{5}$ If $M$ is two-dimensional, the completion of $\operatorname{Diff}_{\mu}(M)$ in the $L^{2}$ metric is unknown. 
In general local (in time) well-posedness refers to constructing a unique solution for a given initial data on a short time interval which depends at least continuously on the data. However it is desirable that the dependence be at least $C^{1}$ smooth. Indeed, in this case applying the inverse function theorem for Banach manifolds, it is possible to deduce that (as in finite dimensions) the exponential map is a local diffeomorphism; this implies in particular nondegeneracy of the geodesic distance as in Section 3.1. Furthermore, other geometric tools such as Jacobi fields and curvature can be introduced to study rigorously stability in the problem of geodesic deviation (we shall elaborate on this in Section 4).

The exponential maps defined on suitable Sobolev completions in the examples discussed so far are either at least $C^{1}$ smooth or else continuous (even differentiable) but not $C^{1}$. The former include, for Sobolev index $s$ sufficiently large,

- the $L^{2}$ metric on the volumorphism group $\operatorname{Diff}_{\mu}^{s}(M)$ whose geodesics correspond to the Euler equations of ideal hydrodynamics; see [14],

- the $H^{1}$ metric on $\operatorname{Diff}_{\mu}^{s}(M)$ corresponding to the Lagrangian-averaged Euler equation; see [64],

- the $H^{1}$ metric on $\operatorname{Diff}^{s}\left(S^{1}\right)$ corresponding to the Camassa-Holm equation in [9] and its generalization to $\operatorname{Diff}^{s}(M)$ and the EPDiff equation in [53],

- the $H^{1}$ metric on the free loop space $\overline{\Omega M}^{H^{1}}$ in [50],

- the homogeneous $\dot{H}^{1}$ metric on $\operatorname{Diff}^{s}\left(S^{1}\right) / S^{1}$ corresponding to the HunterSaxton equation in [32] and its generalization (2.15) on $\operatorname{Diff}^{s}(M) / \operatorname{Diff}_{\mu}^{s}(M)$; see [31],

- the right-invariant $L^{2}$ metrics on $\operatorname{Met}^{s}(M), \operatorname{Met}_{\mu}^{s}(M)$ and $\operatorname{Vol}^{s}(M)$ in $[11,19]$,

- the noninvariant $L^{2}$ metric on $H^{s}(N, M)$ or Diffs $(M)$ whose geodesics are described by pointwise geodesics on $M$; see [14].

The metrics for which $C^{1}$ dependence fails include

- the $L^{2}$ metric on the Virasoro group whose geodesic equation corresponds to the Korteweg-de Vries equation; see [10],

- the right-invariant $L^{2}$ metric on $\operatorname{Diff}^{s}\left(S^{1}\right)$ which yields the (right-invariant) Burgers equation [9] or its higher-dimensional generalization and the template-matching equation [45], 
- the $L^{2}$ metric on unit-parametrized curves in the plane yielding the whip equation (2.13) in [61], or on the equivalence classes of curves under reparametrizations in [46].

Later on we will describe examples where the exponential map fails to be $C^{1}$ as a result of accumulation of conjugate points at $t=0$ (as in [9], [10], [49], and [61]); this is the most common way to prove the result.

It is well-known that the group of volumorphisms $\operatorname{Diff}_{\mu}^{s}(M)$ of a two-dimensional manifold $M$ equipped with the right-invariant $L^{2}$ metric is geodesically complete, i.e., its geodesics which correspond to solutions of the 2D incompressible Euler equations are defined globally in time when $s>2 .{ }^{6}$ This result is due to Wolibner [71] with subsequent contributions by Yudovich [72] and Kato [28] and follows from conservation of vorticity, although the argument is not routine. In three dimensions the problem is open and challenging.

One might expect the right-invariant $H^{1}$ metric on the volumorphism group to be somewhat better behaved, but as of now we have the same result: global existence in two dimensions is relatively easy [64] but in three dimensions is unknown [26]. On the other hand, it is known that smooth solutions of the onedimensional Camassa-Holm equation (2.8) break down for certain initial data $[43,44]$. All solutions of the periodic Hunter-Saxton equation (2.14) as well as its higher-dimensional generalization (2.15) are also known to blow up in finite time.

For the non-invariant $L^{2}$ metric on $\operatorname{Diff}^{s}(M)$ (whose geodesics are given by pointwise geodesics on $M$ ) global existence clearly fails: for a typical initial velocity field two geodesics will eventually cross (which corresponds to a "shock"). On the other hand in $H^{s}(M, M)$ geodesics exists for all time, since such maps need not be injective.

Geodesics in the space of metrics $\operatorname{Met}^{s}(M)$ and volume forms $\operatorname{Vol}^{s}(M)$ typically become degenerate in finite time (see [8] and [19]), while geodesics in $\operatorname{Met}_{\mu}^{s}(M)$ persist for all time [11]. Both the $L^{2}$ and $H^{1}$ metrics on the homogeneous space of equivalence classes of curves in the plane admit geodesics that degenerate

${ }^{6}$ We do not discuss weak solutions (for which much of the geometry seems to break down) of the PDE mentioned above. 
to points [46]. On the space of unit-speed curves, the whip equation would be expected to blow up in finite time physically [61], but this is not yet proved.

\section{JaCobi Fields, CURVATURe, AND STABILITY}

As mentioned in the Introduction, one application of Riemannian techniques in the study of equations of fluid dynamics has been to the problem of (Lagrangian) stability using the equation of geodesic deviation (the Jacobi equation), which involves the curvature tensor. In this section we describe this approach for a general infinite-dimensional manifold equipped with a possibly weak Riemannian metric, but whose exponential map is assumed to be at least $C^{1}$. We will discuss Jacobi fields (as infinitesimal perturbations) and the role played by sectional curvature and its sign. Various results for specific examples mentioned in the previous sections will be the subject of Section 5 .

Let $\mathcal{M}$ be a (possibly weak) Riemannian Hilbert manifold whose geodesic equation is written in the form

$$
\frac{D}{d t} \frac{d \gamma}{d t}=0
$$

where $\frac{d \gamma}{d t}$ is the tangent vector field and $\frac{D}{d t}$ is the covariant derivative along the curve $\gamma(t)$ in $\mathcal{M}$. If $\bar{\gamma}(s, t)$ is a family of geodesics with $\bar{\gamma}(0, t)=\gamma(t)$ then the formula

$$
J(t)=\frac{\partial \bar{\gamma}}{\partial s}(0, t)
$$

gives a Jacobi field $J(t)$ along $\gamma$, i.e., a solution of the Jacobi equation

$$
\frac{D^{2} J}{d t^{2}}+R\left(J, \frac{d \gamma}{d t}\right) \frac{d \gamma}{d t}=0
$$

obtained by differentiating the geodesic equation in $s$ and evaluating at $s=0$. As in finite dimensions, the Riemann curvature tensor $R$ of $\mathcal{M}$ arises here due to the fact that covariant derivatives do not commute in general. Furthermore, the basic result of Cartan applies as well, so that for any $v$ and $w \in T_{p} \mathcal{M}$ we have

$$
\left(D \exp _{p}\right)_{t v}(t w)=J(t)
$$

where $J(t)$ is the Jacobi field along $\gamma(t)=\exp _{p}(t v)$ solving (4.2) with initial conditions $J(0)=0$ and $J^{\prime}(0)=w$. 
Recall that the sectional curvature in the direction of the 2-plane $\sigma$ spanned by the vectors $X_{p}, Y_{p} \in T_{p} \mathcal{M}$ is given by the formula

$$
K_{p}(\sigma)=\frac{\left\langle R\left(X_{p}, Y_{p}\right) Y_{p}, X_{p}\right\rangle}{\left|X_{p}\right|^{2}\left|Y_{p}\right|^{2}-\left\langle X_{p}, Y_{p}\right\rangle} .
$$

Example 4.1. On a two-dimensional Riemannian manifold, the Jacobi equation can be reduced to a single ODE for a function $j(t)$ representing the component of $J$ orthogonal to $\dot{\gamma}$, which takes the form

$$
\frac{d^{2} j(t)}{d t^{2}}+K_{\gamma(t)} j(t)=0
$$

where $K$ is the sectional curvature at a point $\gamma(t)$. In the special case where $K$ is constant, the solution of (4.5) with $j(0)=0$ is

$$
j(t)=j^{\prime}(0) \cdot \begin{cases}\frac{1}{\sqrt{K}} \sin \sqrt{K} t & K>0 \\ t & K=0 . \\ \frac{1}{\sqrt{|K|}} \sinh \sqrt{|K|} t & K<0\end{cases}
$$

This simple special case is the source of much of our intuition about curvature and stability. Suppose that we know precisely the initial position of a particle traveling along a geodesic and its initial velocity only approximately. If $K>0$ then all Jacobi fields are bounded uniformly in time, and thus geodesics starting with nearby initial velocities will remain nearby for all time. If $K<0$ then the Jacobi fields grow exponentially in time, so that small errors are magnified and the motion is unpredictable. If $K=0$ then the growth is linear. On higher dimensional manifolds with variable curvature things become more subtle.

Recall that singular values of the Riemannian exponential map are called conjugate points. More precisely, two points $p$ and $q$ along a geodesic in $\mathcal{M}$ are conjugate if $D \exp _{p}$, viewed as a linear operator from $T_{p} \mathcal{M}$ to $T_{q} \mathcal{M}$ given by (4.3), either fails to be injective (in which case the points are called mono-conjugate) or it fails to be surjective (the points are called epi-conjugate); see Grossman [21]. In finite dimensions both types coincide.

Next, we state the Rauch comparison theorem for weak Riemannian metrics following Biliotti [6]. This result relates growth of Jacobi fields to bounds on the sectional curvature and is a far-reaching generalization of Sturm's comparison theorem on oscillation of solutions of second order ODE. 
Theorem 4.2. Let $\mathcal{M}$ and $\tilde{\mathcal{M}}$ be (possibly infinite-dimensional) weak Riemannian manifolds modeled on Hilbert spaces $E$ and $\tilde{E}$, with $E$ isometric to a closed subspace of $\tilde{E}$. Assume that $\mathcal{M}$ and $\tilde{\mathcal{M}}$ have smooth Levi-Civita connections (and hence smooth exponential maps) with sectional curvatures $K$ and $\tilde{K}$. Let $\gamma$ and $\tilde{\gamma}$ be two geodesics of equal length and suppose that for every $X \in T_{\gamma(t)} \mathcal{M}$ and $\tilde{X} \in T_{\tilde{\gamma}(t)} \tilde{\mathcal{M}}$ we have

$$
K\left(X, \gamma^{\prime}(t)\right) \leq \tilde{K}\left(\tilde{X}, \tilde{\gamma}^{\prime}(t)\right)
$$

Let $J$ and $\tilde{J}$ be the Jacobi fields along $\gamma$ and $\tilde{\gamma}$ such that

- $J(0)=0$ and $\tilde{J}(0)=0$,

- $J^{\prime}(0)$ is orthogonal to $\gamma^{\prime}(0)$ and $\tilde{J}^{\prime}(0)$ is orthogonal to $\tilde{\gamma}^{\prime}(0)$, and

- $\left\|J^{\prime}(0)\right\|=\left\|\tilde{J}^{\prime}(0)\right\|$.

If $\tilde{J}(t)$ is nowhere zero in the interval $(0, a]$ and if $\tilde{\gamma}$ has at most a finite number of points which are epi-conjugate but not mono-conjugate in $(0, a]$, then

$$
\|J(t)\| \geq\|\tilde{J}(t)\| \quad \text { for all } t \in[0, a] .
$$

It often happens that such pathological points which are epi-conjugate but not mono-conjugate can fill out a whole interval (this is the case for the volumorphism group of a three-dimensional manifold [60]) so that the criterion above may only be useful if the exponential map is Fredholm; see Remark 4.6 below.

Remark 4.3. Theorem 4.2 implies that if $\tilde{K}(X, \dot{\gamma}) \geq-k$ for some positive constant $k$ (e.g., take $M$ to be a constant negative curvature space) and if $\tilde{\gamma}$ is free of conjugate points, then any Jacobi field along $\tilde{\gamma}$ in $\tilde{M}$ satisfies $\|\tilde{J}(t)\| \leq$ $\left\|J^{\prime}(0)\right\| k^{-1 / 2} \sinh k t$, which gives essentially the maximum Lyapunov exponent for the system. In the opposite direction, if $K(X, \dot{\gamma}) \leq 0$ (i.e., take $\tilde{M}$ to be a flat space) then $\|J(t)\| \geq\left\|J^{\prime}(0)\right\| t$, which can be interpreted as a weak instability with perturbations growing at least linearly in time. In general, however, one should be cautious when drawing conclusions based on the Rauch theorem and one's finite-dimensional intuition: positive curvature does not imply stability, while negative curvature does not necessarily imply exponential instability, as we discuss below.

For a general Riemannian manifold without additional structure one does not expect more precise results on the relation between curvature and stability. However, most of our examples have a group structure under which the Riemannian 
metric is right-invariant, which can be used to get additional information. To this end it will be convenient to decouple the Jacobi equation (4.2) into two first-order equations.

Namely, let $\mathcal{M}$ be a group $G$ with a right-invariant (weak) Riemannian metric. As in Section 2.1 its geodesics $\gamma(t)$ can be described by a pair of equations consisting of the Euler-Arnold equation (2.1) and the flow equation (2.3)

$$
\frac{d \gamma}{d t}=D R_{\gamma}(u), \quad \frac{d u}{d t}+\operatorname{ad}_{u}^{*} u=0
$$

defined in $G$ and $T_{e} G$ respectively. Let $\bar{\gamma}(s, t)$ be a family of such geodesics with $\bar{\gamma}(0, t)=\gamma(t)$ and with Eulerian velocity $\bar{u}=D R_{\bar{\gamma}^{-1}} \frac{d \bar{\gamma}}{d t}$. Setting $y=D R_{\gamma^{-1}} J$ (where $J$ is the Jacobi field along $\gamma$ as in (4.1)) and $z=\left.\frac{\partial \bar{u}}{\partial s}\right|_{s=0}$ and differentiating both equations in (4.7) with respect to $s$ at $s=0$, we obtain a splitting of the Jacobi equation (4.2) into

$$
\begin{aligned}
& \frac{d y}{d t}-\operatorname{ad}_{u} y=z \\
& \frac{d z}{d t}+\operatorname{ad}_{u}^{*} z+\operatorname{ad}_{z}^{*} u=0 .
\end{aligned}
$$

The linearized Euler equation (4.9) can be used to define a notion of stability: a solution $u$ of the Euler equation is (linearly) stable if every perturbation $z$ is bounded uniformly in time. Using (4.8) one can then relate this notion to stability of Lagrangian trajectories (as in [57] for the volumorphism group) and draw sharper conclusions about the behaviour of geodesics than is generally possible using only Rauch's theorem. The next two examples illustrate the subtleties.

Example 4.4 (Rigid body motion). Let $G=S O(3)$. Its Lie algebra $\mathfrak{s o}(3)=T_{e} G$ is spanned by the vectors $e_{1}, e_{2}$ and $e_{3}$ satisfying $\left[e_{1}, e_{2}\right]=e_{3},\left[e_{2}, e_{3}\right]=e_{1}$, $\left[e_{3}, e_{1}\right]=e_{2}$. This is the group of antisymmetric matrices represented as

$$
x^{1} e_{1}+x^{2} e_{2}+x^{3} e_{3}=\left(\begin{array}{ccc}
0 & -x^{1} & -x^{2} \\
x^{1} & 0 & -x^{3} \\
x^{2} & x^{3} & 0
\end{array}\right) .
$$

The shape of the rigid body determines a left-invariant ${ }^{7}$ Riemannian metric by the conditions $\left\langle e_{1}, e_{1}\right\rangle=\lambda_{1},\left\langle e_{2}, e_{2}\right\rangle=\lambda_{2}$, and $\left\langle e_{3}, e_{3}\right\rangle=\lambda_{3}$ for some positive

\footnotetext{
7 All of our equations so far which have been stated for right-invariant metrics apply to leftinvariant metrics after a possible change of the sign.
} 
numbers $\lambda_{1}, \lambda_{2}, \lambda_{3}$. The left-invariant analogue of (4.7) reads as follows

$$
\frac{d \gamma}{d t}=\gamma u, \quad \frac{d u}{d t}=\operatorname{ad}_{u}^{*} u
$$

with the Euler-Arnold equation given explicitly by

$$
\frac{d u^{1}}{d t}=\frac{\lambda_{2}-\lambda_{3}}{\lambda_{1}} u^{2} u^{3}, \quad \frac{d u^{2}}{d t}=\frac{\lambda_{3}-\lambda_{1}}{\lambda_{2}} u^{1} u^{3}, \quad \frac{d u^{3}}{d t}=\frac{\lambda_{1}-\lambda_{2}}{\lambda_{3}} u^{1} u^{2} .
$$

Consider one steady solution of this equation given by $u^{1}=u^{3}=0$ with $u^{2}=1$, supposing that $0<\lambda_{1}<\lambda_{2}<\lambda_{3}$. The linearized Euler equation (4.9) takes the form

$$
\frac{d z^{1}}{d t}=\frac{\lambda_{2}-\lambda_{3}}{\lambda_{1}} z^{3}, \quad \frac{d z^{2}}{d t}=0, \quad \frac{d z^{3}}{d t}=\frac{\lambda_{1}-\lambda_{2}}{\lambda_{3}} z^{1}
$$

and if $\lambda_{1}<\lambda_{2}<\lambda_{3}$ it admits exponentially growing solutions. ${ }^{8}$ The linearized flow equation (4.8) takes the form

$$
\frac{d y^{1}}{d t}+y^{3}=z^{1}, \quad \frac{d y^{2}}{d t}=z^{2}, \quad \frac{d y^{3}}{d t}-y^{1}=z^{3},
$$

so that $y(t)$ grows exponentially if $z(t)$ does. On the other hand, sectional curvature in all directions containing $e_{2}$ can be made positive by a suitable choice of $\lambda$ 's. Using e.g., the formulas of Milnor [47] we compute for $x=x^{1} e_{1}+x^{2} e_{2}$ that

$$
\begin{array}{rl}
\left\langle R\left(e_{2}, x\right) x, e_{2}\right\rangle=\frac{\left(\lambda_{2}-\lambda_{1}\right)^{2}-}{\lambda_{3}^{2}+2 \lambda_{3}\left(\lambda_{2}-\lambda_{3}+\lambda_{1}\right)}\left(x^{1}\right)^{2} & 4 \lambda_{3} \\
& +\frac{\left(\lambda_{3}-\lambda_{2}\right)^{2}-\lambda_{1}^{2}+2 \lambda_{1}\left(\lambda_{1}-\lambda_{3}-\lambda_{2}\right)}{4 \lambda_{1}}\left(x^{3}\right)^{2},
\end{array}
$$

which is positive-definite if e.g., $\lambda_{1}=\frac{4}{5}, \lambda_{2}=1, \lambda_{3}=\frac{6}{5}$. Hence, we have positive curvature along the geodesic but exponentially growing Jacobi fields. This happens because the Rauch comparison theorem bounds Jacobi fields only up to the first conjugate point; beyond that point all bets are off.

Example 4.5 (Couette flow). Consider $\mathcal{M}=\operatorname{Diff}_{\mu}\left([0,1] \times S^{1}\right)$ with the $L^{2}$ metric. The "plane-parallel Couette flow" $u(x, y)=x \frac{\partial}{\partial y}$ is a steady solution of the Euler equation. This solution is known to be Eulerian stable $[2,56]$ even though the sectional curvature is non-positive in all sections and typically negative [48]. A closer inspection reveals that the growth of all Jacobi fields is precisely linear [57]. Hence, we do no better than what Rauch's theorem says: negative curvature need not imply exponential instability.

${ }^{8}$ This corresponds to the well-known fact that rotation of a rigid body about its largest and smallest axes is stable, but rotation about the middle axis is unstable. 
It is worth pointing out that one can relate Eulerian stability to Lagrangian stretching, i.e., to the growth of $\operatorname{Ad}_{\eta(t)}$ in the operator norm. Using the formula $d\left(\operatorname{Ad}_{\eta} y\right) / d t=\operatorname{Ad}_{\eta}\left(d y / d t-\operatorname{ad}_{u} y\right)$ and defining $Y$ and $Z$ by $y=\operatorname{Ad}_{\eta} Y$ and $z=\operatorname{Ad}_{\eta} Z$, equation (4.8) can be rewritten as

$$
\frac{d Y}{d t}=Z
$$

while equation (4.9) becomes

$$
\frac{d}{d t}\left(\operatorname{Ad}_{\eta}^{*} \operatorname{Ad}_{\eta} Z\right)+\operatorname{ad}_{Z}^{*} u_{0}=0
$$

after incorporating conservation of vorticity $\operatorname{Ad}_{\eta}^{*} u=u_{0}$; see [53]. Observe that the operator $Z \rightarrow \operatorname{Ad}_{\eta(t)}^{*} \operatorname{Ad}_{\eta(t)} Z$ is selfadjoint and positive-definite, while $Z \mapsto \operatorname{ad}_{Z}^{*} u_{0}$ is anti-selfadjoint with constant coefficients. This makes (4.11) somewhat simpler to analyze than (4.9) (even if $u$ is independent of time) because $z \mapsto \operatorname{ad}_{u}^{*} z+\operatorname{ad}_{z}^{*} u$ is not selfadjoint; see [66].

Remark 4.6 (Fredholm exponential maps and conjugate points). If the exponential map of a weak Riemannian manifold $\mathcal{M}$ is known to be smooth, one can ask about the distribution and nature of its singular values (conjugate points): can mono-conjugate and epi-conjugate points coincide, have finite multiplicity, or be discretely distributed along finite geodesic segments?

These questions turn out to have positive answers if the derivative of the exponential map is a Fredholm operator between the tangent spaces of $\mathcal{M}$ with index zero. ${ }^{9}$ This was first established for the free loop space $\overline{\Omega M^{H}}$ with the Sobolev $H^{1}$ metric (2.12) in [50]. In this case the proof of Fredholmness is based on the fact that the curvature operator $R$ in (4.2) is compact. In general one does not expect compactness of the curvature operator. However, in the special case when $\mathcal{M}$ is a group $G$ with a right-invariant metric, one can analyze the derivative of the exponential map using the pair of equations (4.10)-(4.11) to conclude that it is a sum of two terms, the first determined by the positivedefinite operator $Z \rightarrow \operatorname{Ad}_{\eta}^{*} \operatorname{Ad}_{\eta} Z$ and the second a composition of a bounded map with $Y \rightarrow \operatorname{ad}_{Y}^{*} u_{0}$. The former is invertible. Thus if the latter is compact, then the exponential map will be Fredholm of index zero. This strategy works on the volumorphism group $\operatorname{Diff}_{\mu}^{s}(M)$ with the $L^{2}$ metric if $n=2$ (but not if $n \geq 3$ )

\footnotetext{
${ }^{9}$ In this case the exponential map is said to be a nonlinear Fredholm map of index zero (provided that $\mathcal{M}$ is connected).
} 
as well as for right-invariant Sobolev metrics of sufficiently high order on any diffeomorphism group; see [53].

Fredholmness fails for the exponential map of the ellipsoid in $\ell^{2}$ with the induced metric (there are sequences of mono-conjugate points accumulating at an epi-conjugate point, or a mono-conjugate point of infinite order [21]). It also fails for the free loop space with the $L^{2}$ metric [50] and for the volumorphism group $\operatorname{Diff}_{\mu}^{s}(M)$ of a three-dimensional manifold in the $L^{2}$ metric [60]. In the latter case, we can have mono-conjugate points that are dense in an interval and epi-conjugate points that fill up an interval.

On the other hand, smoothness of the exponential map implies (by the inverse function theorem) that any sufficiently short geodesic segment is free of conjugate points and hence is locally minimizing. In particular, if for some $t_{n} \searrow 0$ the points $\gamma\left(t_{n}\right)$ along a given geodesic are mono-conjugate to $\gamma(0)$, then the exponential is not $C^{1}$. This method was used to prove that the exponential maps associated to the KdV equation [49], the right-invariant Burgers equation [9], and the whip equation (2.13) [61] cannot be $C^{1}$.

\section{The Sign of the Curvature: Previous Results}

In the remainder of the paper we will focus on the sign of the sectional curvature in the examples described above. It turns out that with few exceptions sectional curvature can be positive or negative depending on the two-dimensional direction. This section contains a survey of known results and techniques. New results will be presented in Sections 6 and 7 .

The simplest curvature formula arises on $\operatorname{Diff}(M) \subset C^{\infty}(M, M)$ equipped with the $L^{2}$ metric (2.16). If $U=u \circ \eta$ and $V=v \circ \eta$ are two vector fields on $\operatorname{Diff}(M)$ where $u, v \in T_{e} \operatorname{Diff}(M)$ then the covariant derivative of the $L^{2}$ metric is computed in terms of the covariant derivative on $M$ as $\left(\nabla_{U} V\right)_{\eta}=\left(\nabla_{u} v\right) \circ \eta$. Therefore, the $L^{2}$ curvature of $\operatorname{Diff}(M)$ is completely determined by the Riemannian curvature of $M$ and can be computed [48] directly from the definition as

$$
\langle\langle R(u \circ \eta, v \circ \eta) v \circ \eta, u \circ \eta\rangle\rangle_{L^{2}}=\int_{M}\langle R(u, v) v, u\rangle \circ \eta d \mu .
$$

A few simple observations can be made based on this formula. If the vector fields $u$ and $v$ have unit $L^{2}$ norms and are chosen to have disjoint supports, then the integral on the right-hand side will be zero. Consequently, the $L^{2}$ curvature of 
$\operatorname{Diff}(M)$ cannot be strictly positive or strictly negative even if $M$ has constant (non-zero) curvature. Furthermore, it is also clear that it can be non-negative (non-positive) if and only if the sectional curvature of $M$ is non-negative (nonpositive).

One can similarly obtain relatively simple expressions for the $L^{2}$ curvature of the volumorphism group $\operatorname{Diff}_{\mu}(M)$ (following [48]) or the free loop space $\Omega M$, as well as the curvature of the unit-speed loops in $\mathbb{R}^{2}$ (see [61]). To do this we use submanifold geometry.

As in finite dimensional geometry, if $\mathcal{N}$ is a submanifold of a (weak) Riemannian manifold $\mathcal{M}$, then the induced Levi-Civita connection on $\mathcal{N}$ is related to that on $\mathcal{M}$ via the second fundamental form

$$
\Pi(U, V)=\nabla_{U}^{\mathcal{M}} V-\nabla_{U}^{\mathcal{N}} V
$$

where $U$ and $V$ are vector fields tangent to $\mathcal{N}$. $\Pi$ is symmetric and tensorial (i.e., its value at any $p \in \mathcal{N}$ depends only on the values $U_{p}$ and $V_{p}$ ), and the curvature of $\mathcal{N}$ can then be computed using the Gauss-Codazzi formula

$$
\begin{aligned}
\left\langle\left\langle R^{\mathcal{N}}(U, V) V, U\right\rangle\right\rangle=\left\langle\left\langle R^{\mathcal{M}}(U, V) V, U\right\rangle\right\rangle & \\
& +\langle\langle\Pi(U, U), \Pi(V, V)\rangle-\langle\langle\Pi(U, V), \Pi(U, V)\rangle\rangle .
\end{aligned}
$$

If $\mathcal{N}=\operatorname{Diff}_{\mu}(M)$ is the volumorphism group and $\mathcal{M}=\operatorname{Diff}(M)$ is the group of all diffeomorphisms with the $L^{2}$ metric, then the corresponding second fundamental form is $\Pi(U, V)=\nabla \Delta^{-1} \operatorname{div}\left(\nabla_{u} v\right) \circ \eta$, where $U=u \circ \eta, V=v \circ \eta$ and where $u$ and $v$ are divergence free vector fields on $M$. The following theorem summarizes the known results in this important case; see [40, 41, 48, 58, 63, 67]

Theorem 5.1. Let $M$ be a compact manifold of dimension $n \geq 2$, possibly with boundary. Consider the volumorphism group $\operatorname{Diff}_{\mu}(M)$ with the $L^{2}$ metric. For any $u \in T_{e} \operatorname{Diff}_{\mu}(M)$ define

$$
K_{\min }(u)=\inf _{v \in T_{e} \operatorname{Diff}_{\mu}(M)} \frac{\langle\langle R(v, u) u, v\rangle\rangle_{L^{2}}}{\|v\|_{L^{2}}^{2}\|u\|_{L^{2}}^{2}-\langle\langle u, v\rangle\rangle_{L^{2}}^{2}}
$$

to be the minimum sectional curvature in directions containing $u$, and similarly define $K_{\max }(u)$ to be the maximum curvature. Then we have

(1) $K_{\min }(u)<0$, unless $u$ is a Killing field in which case $K_{\min }(u)=0$;

(2) if $n \geq 3$ and $M$ is flat then $K_{\max }(u)>0$, unless $\operatorname{div}\left(\nabla_{u} u\right)=0$ in which case $K_{\max }(u)=0$; 
(3) if $n=2$ and $M$ is flat then $K_{\max }(u)>0$, unless $u$ is plane parallel $u=f(x) \frac{\partial}{\partial y}$ or purely rotational $u=f(r) \frac{\partial}{\partial \theta}$, in either of which cases $K_{\max }(u)=0$.

Proof. All three statements follow from the Gauss-Codazzi formula (5.2) which is the most effective way to determine the sign of the curvature of $\operatorname{Diff}_{\mu}(M)$. Part (1) can be found in [63] when $n=3$ and $M$ is flat although the technique works in any dimension. In part (2), the fact that $\operatorname{div}\left(\nabla_{u} u\right)=0$ implies $K_{\max }(u)=$ 0 is essentially due to [48] while the converse can be proved using the same approximation scheme as in [59] (used there to find conjugate points). Finally, part (3) is a special case of a result in [58] which works for any steady flow on a surface.

In particular, it follows from Theorem 5.1 that for any $M$ of dimension $n \geq 2$ the $L^{2}$ sectional curvature of $\operatorname{Diff}_{\mu}(M)$ assumes both signs. The first examples in the special case of the flat 2-torus $M=\mathbb{T}^{2}$ were worked out by Arnold [1], who used the Lie-theoretic approach of Section 2.1. He derived a general formula for the sectional curvature of a group $\mathcal{M}=G$ with a right-invariant metric in terms of the coadjoint operator $(2.2)$ as

$$
\begin{aligned}
\langle\langle R(u, v) v, u\rangle\rangle=\frac{1}{4} \| \operatorname{ad}_{v}^{*} u & +\operatorname{ad}_{u}^{*} v \|^{2}-\left\langle\left\langle\operatorname{ad}_{u}^{*} u, \operatorname{ad}_{v}^{*} v\right\rangle\right\rangle \\
& -\frac{3}{4}\left\|\operatorname{ad}_{u} v\right\|^{2}+\frac{1}{2}\left\langle\left\langle\operatorname{ad}_{u} v, \operatorname{ad}_{v}^{*} u-\operatorname{ad}_{u}^{*} v\right\rangle\right\rangle
\end{aligned}
$$

and applied it to the case of $\operatorname{Diff}_{\mu}\left(\mathbb{T}^{2}\right)$.

Example 5.2. If $u$ and $v$ are vector fields on $\mathbb{T}^{2}$ with the stream functions $f(x, y)=\cos (j x+k y)$ and $g(x, y)=\cos (l x+m y)$ then the (unnormalized) sectional curvature of $\operatorname{Diff}_{\mu}\left(\mathbb{T}^{2}\right)$ is

$$
\langle\langle R(u, v) v, u\rangle\rangle_{L^{2}}=-\frac{\pi^{2}(j m-k l)^{4}\left(j^{2}+k^{2}+l^{2}+m^{2}\right)}{\left((j+l)^{2}+(k+m)^{2}\right)\left((j-l)^{2}+(k-m)^{2}\right)}<0 .
$$

On the other hand, if we pick $f(x, y)=\cos (3 k x-y)+\cos (3 k x+2 y)$ and $g(x, y)=\cos (k x+y)+\cos (k x-2 y)$ then

$$
\lim _{k \rightarrow \infty} K(u, v)=\frac{9}{8 \pi^{2}} .
$$

Thus it is easy to find negative curvature, but there are many sections with positive curvature as well. 
There are two cases in which the curvature is known to have a remarkably simple form. The first is $\operatorname{Diff}\left(S^{1}\right)$ with the right-invariant $L^{2}$ metric (that is, $a=1$ and $b=0$ in (1.2)) whose curvature at the identity (and hence everywhere by right invariance) is given by

$$
\langle\langle R(u, v) v, u\rangle\rangle_{L^{2}}=\int_{S^{1}}\left(u v_{x}-v u_{x}\right)^{2} d x
$$

and thus is non-negative. We will show in Section 7 that this attractive formula does not generalize to higher dimensions, and the sectional curvature of the rightinvariant $L^{2}$ metric on $\operatorname{Diff}(M)$ can assume both signs.

The second case is the space of densities $\operatorname{Diff}(M) / \operatorname{Diff}_{\mu}(M)$ equipped with the homogeneous Sobolev $\dot{H}^{1}$ metric obtained by setting $b=1$ and $a=c=0$ in (1.1). This space turns out to be isometric to the round sphere of radius 2 and therefore has constant positive curvature

$$
\langle\langle R(u, v) v, u\rangle\rangle_{\dot{H}^{1}}=\frac{1}{4}\left(\|u\|_{\dot{H}^{1}}^{2}\|v\|_{\dot{H}^{1}}^{2}-\langle\langle u, v\rangle\rangle_{\dot{H}^{1}}^{2}\right) .
$$

We refer to $[36,31]$ for detailed calculations.

When the exponential map is not smooth, the curvature may be positive but unbounded above; this allows for conjugate points that occur arbitrarily close to any given point along a geodesic. In such situations one cannot determine stability studying geodesic deviation even for short times, since the Rauch Theorem 4.2 immediately fails. This applies for example to the right-invariant $L^{2}$ metric on the Virasoro group (which yields the KdV equation) whose exponential map is also known not to be smooth and whose sectional curvature is unbounded and of both signs $[49,10]$.

\section{The sign of the Curvature: the one Dimensional CaSe}

In this section we present new results on the sign of the sectional curvature of the right-invariant $H^{1}$ metric on the group $\operatorname{Diff}\left(S^{1}\right)$. In this case the Sobolev $H^{1}$-metric (1.1) reduces to the $a-b$ metric

$$
\langle\langle u, v\rangle\rangle_{H^{1}}=\int_{0}^{1}\left(a u v+b u_{x} v_{x}\right) d x
$$

with $a>0$ and $b>0$. The corresponding Euler-Arnold equation (2.1) reads

$$
m_{t}=-3 a u u_{x}+b\left(2 u_{x} u_{x x}+u u_{x x x}\right), \quad m=A u=a u-b u_{x x} .
$$


For $a=b=1$ we get the periodic Camassa-Holm equation with period 1. For other values we can rescale by $y=x \sqrt{\frac{a}{b}}$ and $s=t \sqrt{\frac{a}{b}}$ so that (6.2) becomes

$$
u_{s}-u_{s y y}+3 u u_{y}-2 u_{y} u_{y y}-u u_{y y y}=0,
$$

which is the Camassa-Holm equation with period $\sqrt{a / b}$.

Recall from Section 5 that the sectional curvature of $\operatorname{Diff}\left(S^{1}\right)$ equipped with the $a$ - $b$ metric (6.1) is already known in the "end-point" cases where either $a=0$ or $b=0$. In the former case, the sectional curvature is positive and constant (see (5.6)), and in the latter it is non-negative (see (5.5)). We will next show that when both $a$ and $b$ are positive, the curvature of $\operatorname{Diff}\left(S^{1}\right)$ can assume both signs.

Remark 6.1. In what follows we will often use the letter $S$ as a shorthand notation for the (non-normalized) sectional curvature $S(u, v)=\langle\langle R(u, v) v, u\rangle\rangle$ if the metric used is clear from the context.

Lemma 6.2. The sectional curvature of $\operatorname{Diff}\left(S^{1}\right)$ endowed with the right-invariant $a-b$ metric (6.1) where $a>0$ and $b>0$ is given by

$$
S(u, v)=\langle\langle R(u, v) v, u\rangle\rangle_{H^{1}}=\langle\langle\Gamma(u, v), \Gamma(u, v)\rangle\rangle_{H^{1}}-\langle\langle\Gamma(u, u), \Gamma(v, v)\rangle\rangle_{H^{1}},
$$

for any $u, v \in T_{e} \operatorname{Diff}\left(S^{1}\right)$, where $\Gamma$ is the Christoffel map defined by

$$
\Gamma(u, v)=A^{-1} \partial_{x}\left(a u v+\frac{b}{2} u_{x} v_{x}\right), \quad A=a-b \partial_{x}^{2} .
$$

Proof. We have $\operatorname{ad}_{v}^{*} u=A^{-1}\left(2 a u v_{x}+a v u_{x}-2 b v_{x} u_{x x}-b v u_{x x x}\right)$ and therefore an easily verified identity

$$
\operatorname{ad}_{v}^{*} u+\operatorname{ad}_{u}^{*} v=\partial_{x}(u v)+2 \Gamma(u, v),
$$

which yields

$$
\operatorname{ad}_{u}^{*} u=\frac{1}{2} \partial_{x}\left(u^{2}\right)+\Gamma(u, u) .
$$

Using the general curvature formula (5.3), we obtain

$$
\langle\langle R(u, v) v, u\rangle\rangle_{H^{1}}=\|\Gamma(u, v)\|_{H^{1}}^{2}-\left\langle\langle\Gamma(u, u), \Gamma(v, v)\rangle_{H^{1}}+L(u, v),\right.
$$

where

$$
\begin{aligned}
L(u, v)=\left\langle\left\langle\Gamma(u, v), \partial_{x}(u v)\right\rangle\right\rangle_{H^{1}} & -\frac{1}{2}\left\langle\left\langle\Gamma(u, u), \partial_{x}\left(v^{2}\right)\right\rangle\right\rangle_{H^{1}}-\frac{1}{2}\left\langle\left\langle\Gamma(v, v), \partial_{x}\left(u^{2}\right)\right\rangle\right\rangle_{H^{1}} \\
& +\frac{1}{4}\left\|\partial_{x}(u v)\right\|_{H^{1}}^{2}-\frac{1}{4}\left\langle\left\langle\partial_{x}\left(u^{2}\right), \partial_{x}\left(v^{2}\right)\right\rangle\right\rangle_{H^{1}} \\
& -\frac{3}{4}\left\|\operatorname{ad}_{u} v\right\|_{H^{1}}^{2}+\frac{1}{2}\left\langle\left\langle\operatorname{ad}_{u} v, \operatorname{ad}_{v}^{*} u-\operatorname{ad}_{u}^{*} v\right\rangle\right\rangle_{H^{1}}
\end{aligned}
$$


and $\operatorname{ad}_{u} v=-v_{x} u+u_{x} v$. Since $\left\langle\left\langle f, A^{-1} g\right\rangle_{H^{1}}=\int_{S^{1}} f g d x\right.$ for any functions $f$ and $g$, we can perform all these computations without ever explicitly evaluating $A^{-1}$.

It turns out that $L(u, v)$ is always zero. We have $L(u, v)=\int_{S^{1}} \Lambda(u, v) d x$, where

$$
\begin{aligned}
\Lambda(u, v)=\partial_{x} & \left(a u v+\frac{b}{2} u_{x} v_{x}\right) \partial_{x}(u v)-\frac{1}{2} \partial_{x}\left(a u^{2}+\frac{b}{2} u_{x}^{2}\right) \partial_{x}\left(v^{2}\right)-\frac{1}{2} \partial_{x}\left(a v^{2}+\frac{b}{2} v_{x}^{2}\right) \partial_{x}\left(u^{2}\right) \\
& +\frac{a}{4}\left[\partial_{x}(u v)\right]^{2}+\frac{b}{4}\left[\partial_{x}^{2}(u v)\right]^{2}-\frac{a}{4}\left[\partial_{x}\left(u^{2}\right)\right]\left[\partial_{x}\left(v^{2}\right)\right]-\frac{b}{4}\left[\partial_{x}^{2}\left(u^{2}\right)\right]\left[\partial_{x}^{2}\left(v^{2}\right)\right] \\
& -\frac{3 a}{4}\left(u v_{x}-v u_{x}\right)^{2}-\frac{3 b}{4}\left(u v_{x x}-v u_{x x}\right)^{2}+\frac{a}{2}\left(v u_{x}-u v_{x}\right)\left(u v_{x}-v u_{x}\right) \\
& +\frac{b}{2}\left(v u_{x}-u v_{x}\right)\left(-2 v_{x} u_{x x}-v u_{x x x}+2 u_{x} v_{x x}+u v_{x x x}\right) .
\end{aligned}
$$

Expanding this and simplifying, we obtain

$$
\Lambda(u, v)=\frac{b}{2} \frac{\partial}{\partial x}\left(-v^{2} u_{x} u_{x x}-u^{2} v_{x} v_{x x}+u v u_{x} v_{x x}+u v v_{x} u_{x x}\right),
$$

which integrates to zero.

The following proposition shows that it is easy to find sections of positive $H^{1}$ curvature on $\operatorname{Diff}\left(S^{1}\right)$ with the $a-b$ metric (6.1). In fact, the curvature is strictly positive along all subspaces spanned by two trigonometric functions.

Theorem 6.3. Consider $\operatorname{Diff}\left(S^{1}\right)$ endowed with the right-invariant $H^{1}$ metric given at the identity by (6.1) with $a>0$ and $b>0$. If $k$ and $l$ are strictly positive distinct integer multiples of $2 \pi$, then

$$
S(\cos k x, \cos l x)=S(\cos k x, \sin l x)=S(\sin k x, \sin l x)=C(k, l)>0,
$$

where $S(u, v)=\left\langle\langle R(u, v) v, u\rangle_{H^{1}}\right.$ and

$$
C(k, l)=\frac{1}{8}\left(\frac{\left(a+\frac{b}{2} k l\right)^{2}}{a+b(k-l)^{2}}(k-l)^{2}+\frac{\left(a-\frac{b}{2} k l\right)^{2}}{a+b(k+l)^{2}}(k+l)^{2}\right) .
$$

Moreover, for $k$ an integer multiple of $2 \pi$, we have

$$
\begin{aligned}
& S(\cos k x, \sin k x)=2 C(k, k)=\frac{\left(a-\frac{b}{2} k^{2}\right)^{2}}{a+4 b k^{2}} k^{2}>0 \quad \text { and } \\
& S(\cos k x, 1)=S(\sin k x, 1)=2 C(k, 0)=\frac{a^{2} k^{2}}{2\left(a+b k^{2}\right)}>0 .
\end{aligned}
$$


Proof. Let $u=\cos k x$ and $v=\cos l x$. From the definition (6.4) of the Christoffel map $\Gamma$ we have

$$
\begin{aligned}
& \Gamma(u, u)=-\frac{2 a k-b k^{3}}{2 a+8 b k^{2}} \sin (2 k x), \quad \Gamma(v, v)=-\frac{2 a l-b l^{3}}{2 a+8 b l^{2}} \sin (2 l x), \\
& \Gamma(u, v)=-\frac{(k+l)(2 a-b k l)}{4 a+4 b(k+l)^{2}} \sin (k+l) x-\frac{(k-l)(2 a+b k l)}{4 a+4 b(k-l)^{2}} \sin (k-l) x .
\end{aligned}
$$

Substituting these into (6.3) gives the formula for $S(\cos k x, \cos l x)$. The other formulas are proved in a similar way.

Sections of negative $H^{1}$ curvature are trickier to find.

Theorem 6.4. For every choice of $a>0$ and $b>0$, there exist velocity fields $u$ and $v$ such that the sectional curvature of $\operatorname{Diff}\left(S^{1}\right)$ endowed with the $a-b$ metric (6.1) is strictly negative, i.e., $S(u, v)=\langle\langle R(u, v) v, u\rangle\rangle_{H^{1}}<0$.

Proof. Set $\alpha=a /\left(4 \pi^{2} b\right)$ and pick

$$
u(x)=\phi+\cos 4 \pi x, \quad v(x)=\sin 2 \pi x,
$$

where

$$
\phi=-\frac{3}{2} \frac{\alpha^{2}-\alpha-2}{\alpha(\alpha+4)}
$$

substitution into (6.3) yields

$$
S(u, v)=\frac{2 b \pi^{4}\left(\alpha^{4}+18 \alpha^{3}+357 \alpha^{2}-20 \alpha-36\right)}{(\alpha+9)(\alpha+4)^{2}} .
$$

Observe that this quantity is negative for $0<\alpha \leq 0.34$.

The second example is constructed differently and works when $\alpha \geq 0.34$. Choose a positive integer $j$ such that $\frac{1}{2} \sqrt{\alpha / 0.34}<j \leq \sqrt{\alpha / 0.34}$ and define $r=\alpha / j^{2}$ so that $0.34 \leq r<1.36$. Set

$$
\psi=\sqrt{-\frac{\left(73 r^{2}-188 r+45\right)(r+16)}{128(r+9)(r-2)^{2}}} .
$$

It is easy to see that this is defined in the range specified above. Set

$$
u(x)=\cos 2 \pi j x+\psi \cos 4 \pi j x \quad \text { and } \quad v(x)=\sin 2 \pi j x+2 \psi \sin 4 \pi j x .
$$

Substituting into (6.3), we obtain

$$
S(u, v)=-\frac{3 \pi^{4} b j^{4}}{64} \frac{P(r)}{(r+9)^{2}(r+4)(r-2)^{2}},
$$


where $P(r)=1435 r^{6}+21940 r^{5}-55074 r^{4}-222512 r^{3}+584323 r^{2}-215364 r+15552$. This quantity $S(u, v)$ is negative for $0.34 \leq r<1.36$, as desired.

Remark 6.5 (The $\mu \mathrm{CH}$ equation). An interesting example of a right-invariant $H^{1}$-type metric on $\operatorname{Diff}\left(S^{1}\right)$ is given at the identity by

$$
\langle\langle u, v\rangle\rangle_{H_{\mu}^{1}}=c \mu(u) \mu(v)+\int_{S^{1}} u^{\prime}(x) v^{\prime}(x) d x,
$$

for any positive constant $c$, where $\mu(u):=\int_{S^{1}} u(x) d x$ is the mean value of the field over the circle. This metric yields yet another integrable evolution equation

$$
u_{t x x}-2 c \mu(u) u_{x}+2 u_{x} u_{x x}+u u_{x x x}=0
$$

as a geodesic equation on the diffeomorphism group which "interpolates" between the Hunter-Saxton and Camassa-Holm equations. (This equation is sometimes called the $\mu \mathrm{HS}$ or $\mu \mathrm{CH}$ equation and was introduced in [30].) The group $\operatorname{Diff}\left(S^{1}\right)$ equipped with the $H_{\mu}^{1}$ metric above admits sections of negative curvature; e.g., $S(u, v)<0$ whenever

$$
u(x)=\frac{3 \pi^{2} k^{2}}{c}+\cos (4 \pi k x), \quad v(x)=\sin (2 \pi k x),
$$

and $k$ is any nonzero integer.

\section{The Sign of the CURVATURE: HigheR Dimensions}

In order to simplify the formulas we present the results for the case when $M$ is the flat torus $\mathbb{T}^{n}=\mathbb{R}^{n} / \mathbb{Z}^{n}$. We recall the general formula for the Sobolev metric (1.1) in the form

$$
\langle\langle u, v\rangle\rangle_{H^{1}}=a \int_{\mathbb{T}^{n}}\langle u, v\rangle d \mu+b \int_{\mathbb{T}^{n}} \delta u^{b} \cdot \delta v^{b} d \mu+c \int_{\mathbb{T}^{n}}\left\langle d u^{b}, d v^{b}\right\rangle d \mu
$$

and observe from (5.6) that when $a=c=0$, then the corresponding sectional curvature of $\operatorname{Diff}\left(\mathbb{T}^{n}\right)$ is strictly positive and constant.

In this section, we show that in the general $a-b-c$ case, the sectional curvature of (7.1) on Diff $\left(\mathbb{T}^{n}\right)$ assumes both signs. The case when at least two of the parameters $a, b, c$ are nonzero is treated in Section 7.1, the case $b=c=0$ is treated in Section 7.2, and the case $a=b=0$ on the subgroup $\operatorname{Diff}_{\mu, \mathrm{ex}}\left(\mathbb{T}^{2}\right)$ is in Section 7.3. We assume $n=2$; all examples discussed below generalize naturally to higher dimensions. 
7.1. The $H^{1}$-metric on $\operatorname{Diff}\left(\mathbb{T}^{n}\right)$ : the EPDiff equation. In the case when all parameters $a, b$ and $c$ of the $H^{1}$ metric (7.1) are strictly positive, the EulerArnold equation (2.1) is a multidimensional generalization of the Camassa-Holm equation. In the special case where $a=b=c=1$ and the manifold is a flat torus $^{10}$ we obtain the EPDiff equation [24].

Theorem 7.1. If $M$ is the flat torus $\mathbb{T}^{n}$ and at least two of the parameters $a, b, c$ are nonzero in (7.1), then the curvature of Diff $\left(\mathbb{T}^{n}\right)$ takes on both signs.

Proof. The formula for the coadjoint operator for the $a-b-c$ metric (7.1) is

$$
\operatorname{ad}_{v}^{*} u=A^{-1}\left((\operatorname{div} v) A u+d\langle A u, v\rangle+\iota_{v} d A u\right),
$$

where $A v=a v^{b}+b d \delta v^{b}+c \delta d v^{b} ;$ see [31].

If we pick $u=f(x) \frac{\partial}{\partial x}$ and $v=g(x) \frac{\partial}{\partial x}$ then (7.2) gives

$$
\operatorname{ad}_{v}^{*} u=\left(a-b \partial_{x}^{2}\right)^{-1}\left(a\left(2 g_{x} f+f_{x} g\right)-b\left(2 g_{x} f_{x x}+g f_{x x x}\right)\right) .
$$

The value of $c$ is irrelevant in this case, since $d u^{b}=d v^{b}=0$. Thus, formula (5.5) yields examples with positive curvature whenever $a \neq 0$. If $a=0$ but $b$ is nonzero, then positive curvature directions exist since this space is isometric to a sphere [36]. Finally, if $a$ and $b$ are both nonzero, then Theorem 6.4 yields examples of negative curvature.

To finish the proof we need negative-curvature examples when $b=0$ with $a$ and $c$ both nonzero, and when $a=0$ while $b$ and $c$ are both nonzero. We will present them for the two-dimensional flat torus $\mathbb{T}^{2}$.

Let $u=f(x) \frac{\partial}{\partial y}$ and $v=g(x) \frac{\partial}{\partial x}$ so that $A u=\left(a f-c f_{x x}\right) \frac{\partial}{\partial y}$ and $A v=$ $\left(a g-b g_{x x}\right) \frac{\partial}{\partial x}$. If $a \neq 0$, then using (7.2) we find

$$
\begin{aligned}
\operatorname{ad}_{u}^{*} u & =\left(a-b \partial_{x}^{2}\right)^{-1}\left(a f f_{x}-c f_{x} f_{x x}\right) \frac{\partial}{\partial x}, \\
\operatorname{ad}_{v}^{*} u & =\left(a-c \partial_{x}^{2}\right)^{-1}\left(a\left(g_{x} f+g f_{x}\right)-c\left(g_{x} f_{x x}+g f_{x x x}\right)\right) \frac{\partial}{\partial y}, \\
\operatorname{ad}_{u}^{*} v & =0 \\
\operatorname{ad}_{v}^{*} v & =\left(a-b \partial_{x}^{2}\right)^{-1}\left(3 a g g_{x}-b\left(2 g_{x} g_{x x}+g g_{x x x}\right)\right) \frac{\partial}{\partial x} .
\end{aligned}
$$

\footnotetext{
${ }^{10}$ In general, EPDiff involves the rough Laplacian $\nabla^{*} \nabla$ rather than the Hodge Laplacian $d \delta+\delta d$; these operators differ by a Ricci curvature term due to the Bochner-Weitzenböck formula. If the manifold is Einstein the EPDiff metric is a special case of (7.1).
} 
We also have $\operatorname{ad}_{u} v=g f_{x} \frac{\partial}{\partial y}$. If $f(x)=g(x)=\sin k x$ for some $k$ which is an integer multiple of $2 \pi$, then it is easy to see that

$$
\begin{aligned}
\operatorname{ad}_{u}^{*} u & =\frac{k\left(a+c k^{2}\right)}{2\left(a+4 b k^{2}\right)} \sin 2 k x \frac{\partial}{\partial x} \\
\operatorname{ad}_{v}^{*} u & =\frac{k\left(a+c k^{2}\right)}{a+4 c k^{2}} \sin 2 k x \frac{\partial}{\partial y} \\
\operatorname{ad}_{v}^{*} v & =\frac{3 k\left(a+b k^{2}\right)}{2\left(a+4 b k^{2}\right)} \sin 2 k x \frac{\partial}{\partial x} \\
\operatorname{ad}_{u} v & =\frac{k}{2} \sin 2 k x \frac{\partial}{\partial y}
\end{aligned}
$$

Note that these formulas are valid if $a=0$ as well, as long as $b \neq 0$ and $c \neq 0$. Indeed, in this case all vectors have to be projected to the orthogonal complement of the harmonic fields, ${ }^{11}$ but since

$$
\int_{0}^{1} \int_{0}^{1} \sin k x d x d y=\int_{0}^{1} \int_{0}^{1} \sin 2 k x d x d y=0
$$

we see that all components are already orthogonal to the harmonic fields.

Substitution into (5.3) now gives the formula

$$
S(u, v)=-\frac{k^{2}\left(7 a^{3}-8 a^{2} b k^{2}+56 a^{2} c k^{2}+44 a c k^{4} b+76 c^{2} k^{4} a+160 c^{2} k^{6} b\right)}{32\left(a+4 c k^{2}\right)\left(a+4 b k^{2}\right)} .
$$

In particular, when $a=0$ then

$$
S(u, v)=-5 c k^{4} / 16
$$

and when $b=0$ we obtain

$$
S(u, v)=-\frac{k^{2}\left(7 a^{2}+56 a c k^{2}+76 c^{2} k^{4}\right)}{32\left(a+4 c k^{2}\right)} .
$$

In either case the sectional curvature is negative for any $k \neq 0$. All these examples work on $\mathbb{T}^{n}$ as well, if $x$ and $y$ denote the first two variables of the coordinate system.

\footnotetext{
${ }^{11} \overline{\text { The metric }(7.1)}$ on Diff( $\left(\mathbb{T}^{2}\right)$ with $a=0$ is degenerate and only defined on the homogeneous space Diff( $\left(\mathbb{T}^{2}\right) / \mathbb{T}^{2}$. Hence everything is only defined modulo harmonic fields on $\mathbb{T}^{2}$.
} 
7.2. The $L^{2}$-metric on $\operatorname{Diff}\left(\mathbb{T}^{n}\right)$ : the Burgers equation. If $b=c=0$, then the formula (7.1) reduces to the $L^{2}$ inner product, and the corresponding geodesic equation (2.1) is the multi-dimensional Burgers equation

$$
u_{t}+\nabla_{u} u+\operatorname{div}(u) u+\frac{1}{2} \nabla\langle u, u\rangle=0,
$$

also called the template matching equation; see [23]. However, in contrast with the one-dimensional case, the curvature of the right-invariant $L^{2}$-metric assumes both signs when $n \geq 2$. For simplicity we only prove the result for $n=2$.

Proposition 7.2. The sectional curvature of Diff $\left(\mathbb{T}^{2}\right)$ equipped with the rightinvariant metric (7.1) with $b=c=0$ is given by the formula (5.5) for any $u=f(x) \frac{\partial}{\partial x}$ and $v=g(x) \frac{\partial}{\partial x}$, in which case $S(u, v) \geq 0$. On the other hand, if $u=\sin (2 \pi x) \frac{\partial}{\partial x}$ and $v=\sin ^{2}(2 \pi x) \frac{\partial}{\partial y}$ then $S(u, v)<0$.

Proof. In this case the operator $\operatorname{ad}_{v}^{*} u$ defined by (2.2) has the form

$$
\operatorname{ad}_{v}^{*} u=u \operatorname{div} v+\left(\iota_{v} d u^{b}\right)^{\sharp}+\nabla\langle u, v\rangle .
$$

Thus, when $u=f(x) \frac{\partial}{\partial x}$ and $v=g(x) \frac{\partial}{\partial x}$ we get $\operatorname{ad}_{v}^{*} u=\left(2 g_{x} f+g f_{x}\right) \frac{\partial}{\partial x}$, which is the same formula as in the one-dimensional case and the first part of the proposition follows.

Furthermore, if $w=g(x) \frac{\partial}{\partial y}$ then we compute

$$
\begin{aligned}
\operatorname{ad}_{w}^{*} u & =0, \\
\operatorname{ad}_{u}^{*} u & =3 f f_{x} \frac{\partial}{\partial x}, \\
\operatorname{ad}_{u}^{*} w & =\left(f_{x} g+f g_{x}\right) \frac{\partial}{\partial y}, \\
\operatorname{ad}_{w}^{*} w & =g g_{x} \frac{\partial}{\partial x} .
\end{aligned}
$$

Combining these formulas with $\operatorname{ad}_{u} w=-f g_{x} \frac{\partial}{\partial y}$ in (5.3), we get

$$
S(u, w)=a \int_{0}^{1}\left(\frac{1}{4} f_{x}^{2} g^{2}-2 f f_{x} g g_{x}\right) d x .
$$

Taking $f(x)=\sin (2 \pi x)$ and $g(x)=\sin ^{2}(2 \pi x)$, we find $S(u, w)=-15 \pi^{2} / 16$.

A similar consideration in the general case can be summarized as the following statement. 
Theorem 7.3. The sectional curvature of $\operatorname{Diff}\left(\mathbb{T}^{n}\right)$ equipped with the right-invariant $L^{2}$ metric (i.e., the $a-b-c$ metric (7.1) with $a=1$ and $b=c=0$ ) assumes both signs.

7.3. The homogeneous $\dot{H}^{1}$-metric on $\operatorname{Diff}_{\mu, \mathbf{e x}}\left(\mathbb{T}^{2}\right)$. Consider next the Lie group Diff $\operatorname{Dex}_{\text {ex }}\left(\mathbb{T}^{2}\right)$ of exact volumorphisms of the flat torus $\mathbb{T}^{2}$, which consists of symplectic diffeomorphisms preserving the center of mass. Its Lie algebra consists of Hamiltonian vector fields $u=\operatorname{sgrad} f$ with $f \in C^{\infty}\left(\mathbb{T}^{2}\right)$. Following Arnold [1] we calculate the sectional curvature of the metric

$$
\langle\langle u, v\rangle\rangle=c \int_{\mathbb{T}^{2}}\left\langle d u^{b}, d u^{b}\right\rangle d \mu,
$$

on this group. It turns out to be just as convenient to work with a more general right-invariant metric given at the identity by

$$
\langle\langle u, v\rangle\rangle=\langle\langle\operatorname{sgrad} f, \operatorname{sgrad} g\rangle\rangle=\int_{\mathbb{T}^{2}} f \Lambda g d \mu,
$$

where the (positive-definite, symmetric) operator $\Lambda$ defining the inner product is given by the formula $\Lambda=\lambda(\Delta)$ for some function $\lambda: \mathbb{R}^{+} \rightarrow \mathbb{R}^{+}$. ${ }^{12}$ For a vector $p \in \mathbb{R}^{2}$, we will write $F(p)=\lambda\left(|p|^{2}\right)$ for convenience. The metric (7.3) corresponds to $\lambda(z)=c z^{2}$ and $\Lambda=c \Delta^{2}$.

Theorem 7.4. Suppose $f(x, y)=\cos (j x+k y)$ and $g(x, y)=\cos (l x+m y)$, where $j, k, l, m$ are integer multiples of $2 \pi$. Set $p=(j, k)$ and $q=(l, m)$ and let $u=\operatorname{sgrad} f$ and $v=\operatorname{sgrad} g$. Then $^{13}$

$$
\begin{aligned}
S(u, v)=\frac{|p \wedge q|^{2}}{8}\left\{\frac{1}{4}(F(p)\right. & -F(q))^{2}\left(\frac{1}{F(p+q)}+\frac{1}{F(p-q)}\right) \\
& \left.-\frac{3}{4}(F(p+q)+F(p-q))+F(p)+F(q)\right\}
\end{aligned}
$$

where $p \wedge q=j m-k l$.

Proof. Recall that sgrad $f=-f_{y} \frac{\partial}{\partial x}+f_{x} \frac{\partial}{\partial y}$, so that if $\{f, g\}=f_{x} g_{y}-f_{y} g_{x}$ denotes the Poisson bracket, then $[\operatorname{sgrad} f, \operatorname{sgrad} g]=\operatorname{sgrad}\{f, g\}$. Given any smooth

12 More precisely, if a function $f$ is written in an eigenbasis of the positive-definite Laplacian as $f=\sum_{k} a_{k} \phi_{k}$ with $\Delta \phi_{k}=\gamma_{k} \phi_{k}$, then $\Lambda f=\sum_{k} a_{k} \lambda\left(\gamma_{k}\right) \phi_{k}$.

${ }^{13}$ Note that for $F(p)=|p|^{2}$ formula (7.5) reproduces (5.4), up to a rescaling factor. 
functions $f, g$ and $h$ on the torus let $u=\operatorname{sgrad} f, v=\operatorname{sgrad} g$ and $w=\operatorname{sgrad} h$ be the corresponding skew-gradients. Integration by parts gives

$$
\begin{aligned}
\left\langle\left\langle\operatorname{ad}_{v}^{*} u, w\right\rangle\right\rangle & =-\int_{\mathbb{T}^{2}}(\Lambda f)\{g, h\} d \mu \\
& =\int_{\mathbb{T}^{2}} h\{g, \Lambda f\} d \mu=\left\langle\left\langle w, \operatorname{sgrad} \Lambda^{-1}\{g, \Lambda f\}\right\rangle,\right.
\end{aligned}
$$

from which we deduce that $\operatorname{ad}_{v}^{*} u=\operatorname{sgrad} \Lambda^{-1}\{g, \Lambda f\}$.

We can assume that $|p|,|q|,|p-q|$, and $|p+q|$ are all nonzero. Furthermore, since $\Delta f=|p|^{2} f$ and $\Delta g=|q|^{2} g$ we find that

$$
\begin{aligned}
\operatorname{ad}_{u}^{*} u & =\operatorname{ad}_{v}^{*} v=0, \\
\operatorname{ad}_{v}^{*} u & =-F(p) \operatorname{sgrad} \Lambda^{-1} \theta, \quad \text { and } \quad \operatorname{ad}_{u}^{*} v=F(q) \operatorname{sgrad} \Lambda^{-1} \theta,
\end{aligned}
$$

where

$$
\begin{aligned}
\theta(x, y)=\{f, g\}(x, y)=\frac{1}{2}(j m-k l)( & \cos ((j-l) x+(k-m) y) \\
& -\cos ((j+l) x+(k+m) y)) .
\end{aligned}
$$

Clearly, we have $\theta=\frac{1}{2}(p \wedge q)(\varphi-\psi)$, where $\varphi$ and $\psi$ are eigenfunctions satisfying $\Lambda \varphi=F(p-q) \varphi$ and $\Lambda \psi=F(p+q) \psi$. Combining the above formulas we obtain

$$
\begin{aligned}
& \operatorname{ad}_{u} v=-\operatorname{sgrad} \theta=-\frac{p \wedge q}{2} \operatorname{sgrad}(\varphi-\psi), \\
& \operatorname{ad}_{v}^{*} u=-\frac{(p \wedge q) F(p)}{2} \operatorname{sgrad}\left(\frac{\varphi}{F(p-q)}-\frac{\psi}{F(p+q)}\right), \\
& \operatorname{ad}_{u}^{*} v=\frac{(p \wedge q) F(q)}{2} \operatorname{sgrad}\left(\frac{\varphi}{F(p-q)}-\frac{\psi}{F(p+q)}\right) .
\end{aligned}
$$

Since, on the other hand, we have

$$
\begin{gathered}
\langle\langle\operatorname{sgrad} \varphi, \operatorname{sgrad} \varphi\rangle\rangle=\frac{1}{2} F(p-q), \quad\langle\langle\operatorname{sgrad} \varphi, \operatorname{sgrad} \psi\rangle\rangle=0, \\
\text { and }\langle\langle\operatorname{sgrad} \psi, \operatorname{sgrad} \psi\rangle\rangle=\frac{1}{2} F(p+q),
\end{gathered}
$$

substituting into the sectional curvature formula (5.3) yields (7.5).

Corollary 7.5. In the particular case when the metric is given by (7.3) the sectional curvature can assume both signs depending on $p$ and $q$. 
Indeed, for the metric (7.3) we have $F(p)=\lambda\left(|p|^{2}\right)=c|p|^{4}$, and a straightforward computation gives $S(u, v)>0$ when $p=(10 \pi, 0)$ and $q=(8 \pi, 2 \pi)$, while $S(u, v)<0$ when $p=(2 \pi, 0)$ and $q=(0,2 \pi)$.

\section{Appendix A. SEmi-DiRECT PROdUCts AND CURVATURE Formulas}

Formula (6.3) for the sectional curvature of the $H^{1}$ metric derived in Lemma 6.2 of Section 6 resembles the formula for the curvature of a Riemannian submanifold $\mathcal{N}$ isometrically immersed in an ambient manifold $\mathcal{M}$, given by (5.2). Observe however that the sign of (5.2) is the opposite of the sign for (6.3). The next proposition shows how to rearrange (6.3) to make the analogy work.

Proposition A.1. The sectional curvature (6.3) of $\operatorname{Diff}\left(S^{1}\right)$ with the $H^{1}$ metric (6.1) can be rewritten in either of the two following forms

$$
S(u, v)=-\frac{a}{2} \int_{S^{1}}\left(u v_{x}-v u_{x}\right)^{2} d x+\frac{a}{b}\langle\langle T(u, u), T(v, v)\rangle\rangle-\frac{a}{b}\langle\langle T(u, v), T(u, v)\rangle\rangle,
$$

$$
\begin{aligned}
S(u, v)=\frac{1}{a} \int_{S^{1}} & \left(a\left(u v_{x}-v u_{x}\right)+\frac{b}{2}\left(v_{x} u_{x x}-u_{x} v_{x x}\right)\right)^{2} d x \\
+ & \frac{b}{a}\langle\langle Q(u, u), Q(v, v)\rangle\rangle-\frac{b}{a}\langle\langle Q(u, v), Q(u, v)\rangle\rangle,
\end{aligned}
$$

where the bilinear operators $T$ and $Q$ are defined by

$$
T(u, v)=A^{-1}\left(a u v+\frac{b}{2} u_{x} v_{x}\right), \quad Q(u, v)=-\partial_{x}^{2} A^{-1}\left(a u v+\frac{b}{2} u_{x} v_{x}\right) .
$$

Proof. Note that $\Gamma(u, v)$ defined by (6.4) is related to $Q$ and $T$ by $\Gamma(u, v)=$ $\partial_{x} T(u, v)$ and $Q(u, v)=-\partial_{x} \Gamma(u, v)$.

We prove (A.1) first. Let $u$ and $v$ be any vector fields and set $q=a u v+\frac{b}{2} u_{x} v_{x}$. Then $T(u, v)=A^{-1} q$, so that

$$
\langle\langle\Gamma(u, v), \Gamma(u, v)\rangle\rangle=\int_{S^{1}} \partial_{x} T(u, v) A \partial_{x} T(u, v) d x=-\int_{S^{1}} q A^{-1} \partial_{x}^{2} q d x .
$$

Now using the identity $A^{-1} \partial_{x}^{2}=-\frac{1}{b}+\frac{a}{b} A^{-1}$, we find

$$
\langle\langle\Gamma(u, v), \Gamma(u, v)\rangle\rangle=\frac{1}{b} \int_{S^{1}}\left(a u v+\frac{b}{2} u_{x} v_{x}\right)^{2} d x-\frac{a}{b}\langle\langle T(u, v), T(u, v)\rangle\rangle .
$$

Using the same trick also on the other term in (6.3) gives (A.1). A similar technique reduces (A.2) to (6.3). 
The formulas (A.1) and (A.2) suggest that $\operatorname{Diff}\left(S^{1}\right)$ with the $a-b$ metric (6.1) can be realized as an isometrically immersed submanifold of some simpler manifold. For (A.2) such an immersion is described in the following theorem.

Theorem A.2. Let $\operatorname{Diff}\left(S^{1}\right) \ltimes C^{\infty}\left(S^{1}\right)$ denote the semidirect product of the diffeomorphism group $\operatorname{Diff}\left(S^{1}\right)$ with $C^{\infty}\left(S^{1}\right)$ with group structure given by

$$
(\eta, F) \cdot(\xi, G)=(\eta \circ \xi, F \circ \xi+G) .
$$

Define a right-invariant Riemannian metric on this group by the $L^{2}$ inner product at the identity

$$
\langle\langle(u, f),(v, g)\rangle\rangle=\int_{S^{1}}(a u v+b f g) d x .
$$

Let $\Upsilon: \operatorname{Diff}\left(S^{1}\right) \rightarrow \operatorname{Diff}\left(S^{1}\right) \ltimes C^{\infty}\left(S^{1}\right)$ denote the map $\Upsilon(\eta)=\left(\eta, \ln \eta_{x}\right)$. Then $\Upsilon$ is an embedding and a group homomorphism and the right-invariant metric induced on $\operatorname{Diff}\left(S^{1}\right)$ by (A.4) is the a-b metric (6.1). Furthermore, the curvature formula (A.2) is the Gauss-Codazzi formula for the embedding $\Upsilon$.

Proof. The Lie algebra for $G=\operatorname{Diff}\left(S^{1}\right) \ltimes C^{\infty}\left(S^{1}\right)$ is the semidirect product $\mathfrak{g}=\operatorname{Vect}\left(S^{1}\right) \ltimes C^{\infty}\left(S^{1}\right)$, which has an interpretation as the space of first-order differential operators $v D+f$ for $v \in$ Vect and $f \in C^{\infty}$. For details on the geometry of the semidirect product see e.g., [42, 70]. For this semi-direct product the adjoint operator has the form

$$
\operatorname{ad}_{(u, f)}(v, g)=\left(-u v_{x}+u_{x} v, v f_{x}-u g_{x}\right),
$$

which implies that the Arnold operator (2.2) is

$$
\operatorname{ad}_{(u, f)}^{*}(v, g)=\left(2 u_{x} v+u v_{x}+\frac{b}{a} g f_{x}, g u_{x}+g_{x} u\right) .
$$

The general curvature formula (5.3) implies, after some simplifications, that the curvature of the semidirect product $\operatorname{Diff}\left(S^{1}\right) \ltimes C^{\infty}\left(S^{1}\right)$ can be written as

$$
\begin{array}{r}
\langle\langle\bar{R}((u, f),(v, g))(v, g),(u, f)\rangle\rangle=a \int_{S^{1}}\left(u v_{x}-v u_{x}+\frac{b}{2 a}\left(g f_{x}-f g_{x}\right)\right)^{2} d x \\
+\frac{b}{4} \int_{S^{1}}\left(g u_{x}-f v_{x}\right)\left(g u_{x}-f v_{x}+8 v f_{x}-8 u g_{x}\right) d x .
\end{array}
$$

Observe that $\Upsilon$ is a group homomorphism:

$$
\Upsilon(\eta \circ \xi)=\left(\eta \circ \xi, \ln \left(\eta_{x} \circ \xi\right)+\ln \left(\xi_{x}\right)\right)=\Upsilon(\eta) \cdot \Upsilon(\xi)
$$


using the chain rule. Smoothness of this map can be proved using Sobolev $H^{s}$ topology. For our purposes it is enough to note that $\Upsilon$ is formally an immersion since at the identity we have

$$
D \Upsilon_{e}(u)=\left(u, u_{x}\right)
$$

which is obviously injective. ${ }^{14}$ Hence the induced metric on $\operatorname{Diff}\left(S^{1}\right)$ is given at the identity by

$$
\langle\langle u, v\rangle\rangle=\langle\langle D \Upsilon(u), D \Upsilon(v)\rangle\rangle=\int_{S^{1}}\left(a u v+b u_{x} v_{x}\right) d x,
$$

which is precisely the $a-b$ metric (6.1).

The orthogonal complement of the image of $D \Upsilon_{e}$ in the metric (A.4) consists of those vectors of the form $\left(\frac{b}{a} h_{x}, h\right)$ for some function $h: S^{1} \rightarrow \mathbb{R}$. We can now compute the second fundamental form (5.1) of the embedding: because of the right-invariance, we have $\langle\langle\Pi(u, u), w\rangle\rangle=\left\langle\left\langle\operatorname{ad}_{u}^{*} u, w\right\rangle\right\rangle$ for $w \in\left(\operatorname{Im} D \Upsilon_{e}\right)^{\perp}$, where $\mathrm{ad}^{*}$ is the operator given in (A.5). We then have

$$
\Pi(u, u)=\left(\frac{b}{a} h_{x}, h\right) \quad \text { where } \quad h=-A^{-1} \partial_{x}^{2}\left(a u^{2}+\frac{b}{2} u_{x}^{2}\right) .
$$

Polarization yields $\Pi(u, v)=\left(\frac{b}{a} Q(u, v)_{x}, Q(u, v)\right)$, where $Q$ is given by (A.3). Substituting (A.6) when $f=u^{\prime}$ and $g=v^{\prime}$, together with the second fundamental form, into (5.2) reproduces (A.2).

We conjecture that there is another embedding-homomorphism of $\operatorname{Diff}\left(S^{1}\right)$ into an infinite-dimensional Lie group with right-invariant metric which reproduces formula (A.1), but we do not know what it might be.

Acknowledgements. B.K. was partially supported by the Simonyi Fund and an NSERC research grant. J.L. acknowledges support from the EPSRC, UK. G.M. was supported in part by the James D. Wolfensohn Fund and Friends of the Institute for Advanced Study grants. S.C.P. was partially supported by an NSF grant.

\footnotetext{
${ }^{14}$ In the interpretation via differential operators, we consider operators of the form $u D+u_{x}=D u$. The homomorphism $D \Upsilon: u D \mapsto D u$ is evidently a homomorphism of Lie algebras Vect $\rightarrow$ Vect $\ltimes C^{\infty}$.
} 


\section{REFERENCES}

[1] V. Arnold, Sur la géometrie différentielle des groupes de Lie de dimension infinie et ses application à l'hydrodynamique des fluides parfaits, Ann. Inst. Fourier (Grenoble) 16 (1966), 319-361.

[2] V. Arnold, Notes on the three-dimensional flow pattern of a perfect fluid in the presence of a small perturbation of the initial velocity field, Appl. Math. and Mech. 36 (1972), no. 2, 255-262.

[3] V. Arnold and B. Khesin, Topological methods in hydrodynamics, Springer, New York 1998.

[4] C.J. Atkin, The Hopf-Rinow theorem is false in infinite dimensions, Bull. London Math. Soc. 7 (1975), 261-266.

[5] M. Bauer, M. Bruveris, P. Harms, and P.W. Michor, Vanishing geodesic distance for the Riemannian metric with geodesic equation the KdV-equation, Ann. Global Anal. Geom. 41 (2012), 461-472.

[6] L. Biliotti, The exponential map of a weak Riemannian Hilbert manifold, Illinois J. Math. 48 (2004), no. 4, 1191-1206.

[7] J. Cheeger and D. Ebin, Comparison theorems in Riemannian geometry, North Holland, Amsterdam, 1975.

[8] B. Clarke, The metric geometry of the manifold of Riemannian metrics over a closed manifold, Calc. Var. Partial Differential Equations 39 (2010), no. 3-4, $533-545$.

[9] A. Constantin and B. Kolev, On the geometric approach to the motion of inertial mechanical systems, J. Phys. A 35 (2002), R51-R79.

[10] A. Constantin, T. Kappeler, B. Kolev and P. Topalov, On geodesic exponential maps of the Virasoro group, Ann. Glob. Anal. Geom. 31 (2007), 155-180.

[11] D.G. Ebin, The manifold of Riemannian metrics, in Proc. Sympos. Pure Math. 15, Amer. Math. Soc., Providence, R.I., 1970.

[12] D.G. Ebin, The equations of motion of a perfect fluid with free boundary are not well posed, Comm. Partial Differential Equations 12 (1987), no. 10, 1175-1201.

[13] D.G. Ebin, Geodesics on the symplectomorphism group, Geom. Funct. Anal. (GAFA) 22 (2012), 202-212.

[14] D.G. Ebin and J. E. Marsden, Groups of diffeomorphisms and the motion of an incompressible fluid, Ann. Math. 92 (1970), 102-163.

[15] D.G. Ebin and S.C. Preston, Riemannian geometry on the contactomorphism group, in progress.

[16] I. Ekeland, The Hopf-Rinow theorem in infinite dimension, J. Differential Geometry 13 (1978), 287-301. 
[17] Ya. Eliashberg and L. Polterovich, Bi-invariant metrics on the group of Hamiltonian diffeomorphisms, Internat. J. Math. 4 (1993), 727-738.

[18] H. Eliasson, Condition (C) and geodesics on Sobolev manifolds, Bull. Amer. Math. Soc. 77 (1971), 1002-1005.

[19] D.S. Freed and D. Groisser, The basic geometry of the manifold of Riemannian metrics and of its quotient by the diffeomorphism group, Michigan Math. J. 36 (1989), 323-344.

[20] S. Friedlander and M.M. Vishik, Dynamo theory, vorticity generation, and exponential stretching, Chaos 1 (1991), no. 2, 198-205.

[21] N. Grossman, Hilbert manifolds without epiconjugate points, Proc. A.M.S. 16 (1965), 1365-1371.

[22] J. Hadamard, Sur la forme des lignes géodésiques à l'infini et sur les geodesiques des surfaces réglées du second ordre, Bull. Soc. Math. France 26 (1898), 195-216.

[23] A.N. Hirani, J.E. Marsden, and J. Arvo, Averaged template matching equations, Lect. Notes Comp. Sci. 2134 (2001), 528-543.

[24] D. Holm, J.E. Marsden, and T.S. Ratiu, The Euler-Poincaré equations and semidirect products with applications to continuum theories, Adv. Math. 137 (1998), $1-81$.

[25] D. Holm and V. Zeitlin, Hamilton's principle for quasigeostrophic motion, Phys. Fluids 10 (1998), 800-806.

[26] T. Hou and C. Li, On global well-posedness of the Lagrangian averaged Euler equations, SIAM J. Math. Anal. 38 (2006), 782-794.

[27] T. Kambe, Geometrical theory of dynamical systems and fluid flows, revised edition, World Scientific Publishing, Hackensack, NJ, 2010.

[28] T. Kato, On classical solutions of the two-dimensional non-stationary Euler equation, Arch. Rational Mech. Anal. 25 (1967), 188-200.

[29] B. Khesin, Dynamics of symplectic fluids and point vortices, Geom. Funct. Anal. (GAFA) 22 (2012), 1444-1459.

[30] B. Khesin, J. Lenells, and G. Misiołek, Generalized Hunter-Saxton equation and the geometry of the group of circle diffeomorphisms, Math. Ann. 342 (2008), 617656 .

[31] B. Khesin, J. Lenells, G. Misiołek and S.C. Preston, Geometry of diffeomorphism groups, complete integrability and geometric statistics, Geom. Funct. Anal. (GAFA) 23 (2013), 334-366.

[32] B. Khesin and G. Misiołek, Euler equations on homogeneous spaces and Virasoro orbits, Adv. Math. 176 (2003), 116-144.

[33] B. Khesin and G. Misiołek, Shock waves for the Burgers equation and curvatures of diffeomorphism groups, Proc. Steklov Inst. Math. 259 (2007), no. 1, 73-81. 
[34] B. Khesin and R. Wendt, The geometry of infinite-dimensional groups, Ergebnisse der Mathematik, vol. 51, Springer, New York 2008.

[35] B. Kolev, Geometric differences between the Burgers and the Camassa-Holm equation, J. Nonlinear Math. Phys. 15 Suppl. 2 (2008), 116-132.

[36] J. Lenells, The Hunter-Saxton equation describes the geodesic flow on a sphere, J. Geom. Phys. 57 (2007), 2049-2064.

[37] J. Lenells, The Hunter-Saxton equation: a geometric approach, SIAM J. Math. Anal. 40 (2008), 266-277.

[38] J. Lenells, Weak geodesic flow and global solutions of the Hunter-Saxton equation, Discrete Contin. Dyn. Syst. 18 (2007), no. 4, 643-656.

[39] H. Lindblad, Well-posedness for the motion of an incompressible liquid with free surface boundary, Ann. Math. 162 (2005), 109-194.

[40] A.M. Lukatsky, Negativity of curvatures of the diffeomorphisms groups for locally Euclidean manifolds, Russ. Math. Surv. 45 (1990), no. 6, 160-161.

[41] A.M. Lukatsky, On the curvature of the diffeomorphisms group, Ann. Global Anal. Geom. 11 (1993), no. 2, 135-140.

[42] J. Marsden, T. Ratiu, and A. Weinstein, Reduction and Hamiltonian structures on duals of semidirect product Lie algebras, Contemp. Math. 28 (1984), Amer. Math. Soc., Providence, RI, 55-100.

[43] H.P. McKean, Breakdown of a shallow water equation, Asian J. Math. 2 no. 4 (1998), 867-874.

[44] H.P. McKean, Fredholm determinants and the Camassa-Holm hierarchy, Comm. Pure Appl. Math. 56 no. 5 (2003), 638-680.

[45] P.W. Michor and D. Mumford, Vanishing geodesic distance on spaces of submanifolds and diffeomorphisms. Doc. Math. 10 (2005), 217245.

[46] P.W. Michor and D. Mumford, Riemannian geometries on spaces of plane curves, J. Eur. Math. Soc. 8 (2006), no. 1, 1-48.

[47] J. Milnor, Curvatures of left invariant metrics on Lie groups, Adv. Math. 21 no. 3 (1976), 293-329.

[48] G. Misiołek, Stability of flows of ideal fluids and the geometry of the group of diffeomorphisms, Indiana Univ. Math. J. 42 (1993), 215-235.

[49] G. Misiołek, Conjugate points in the Bott-Virasoro group and the KdV equation, Proc. Amer. Math. Soc. 125 (1997), 935-940

[50] G. Misiołek, The exponential map on the free loop space is Fredholm, Geom. Funct. Anal. 7 (1997), 954-969.

[51] G. Misiołek, A shallow water equation as a geodesic flow on the Bott-Virasoro group, J. Geom. Phys. 24 (1998), 203-208. 
[52] G. Misiołek, Classical solutions of the periodic Camassa-Holm equation, Geom. Funct. Anal. 12 (2002), 1080-1104.

[53] G. Misiołek and S.C. Preston, Fredholm properties of Riemannian exponential maps on diffeomorphism groups, Invent. Math. 179 (2010), no. 1, 191-227.

[54] D. Mumford, Pattern theory and vision, Questions mathématiques en traitement du signal et de l'image, Institute Henri Poincaré, Paris (1998), 7-13.

[55] V. Ovsienko and B. Khesin, The (super) KdV equation as an Euler equation, Funct. Anal. Appl. 21:4 (1987), 81-82.

[56] W. Orr, The stability or instability of the steady motion of a perfect fluid and of a viscous fluid, part I: A perfect fluid, Proc. Roy. Irish Acad. 27 (1907), 9-68.

[57] S.C. Preston, For ideal fluids, Eulerian and Lagrangian instabilities are equivalent, Geom. Func. Anal. (GAFA) 14 no. 5, (2004), 1044-1062.

[58] S.C. Preston, Nonpositive curvature on the area-preserving diffeomorphism group, J. Geom. Phys. 53 (2005), 226-248.

[59] S.C. Preston, On the volumorphism group, the first conjugate point is always the hardest, Comm. Math. Phys. 267 (2006), 493-513.

[60] S.C. Preston, The WKB method for conjugate points in the volumorphism group, Indiana Univ. Math. J. 57 no. 7 (2008), 3303-3328.

[61] S.C. Preston, The geometry of whips, Ann. Global Anal. Geom. 41 (2012), 281305.

[62] S. Rosenberg, The Laplacian on a Riemannian manifold. An introduction to analysis on manifolds, Cambridge University Press, Cambridge, 1997.

[63] P. Rouchon, The Jacobi equation, Riemannian curvature and the motion of a perfect incompressible fluid, European J. Mech. B Fluids 11 (1992), no. 3, 317336 .

[64] S. Shkoller, Geometry and curvature of diffeomorphism groups with $H^{1}$ metric and mean hydrodynamics, J. Funct. Anal. 160 (1998), no. 1, 337-365.

[65] A. Shnirelman, Generalized fluid flows, their approximation and applications, Geom. Funct. Anal. 4 (1994), no. 5, 586-620.

[66] R. Shvydkoy and M. Vishik, On spectrum of the linearized 3D Euler equation, Dyn. Partial Differ. Equ. 1 (2004) no. 1, 49-63.

[67] N.K. Smolentsev, Diffeomorphism groups of compact manifolds, J. Math. Sci. 146 (2007) no. 6, 6213-6312.

[68] J. L. Synge, On the geometry of dynamics, Phil. Trans. Royal Soc. London 226 (1927), 31-106.

[69] F. Tiğlay and C. Vizman, Generalized Euler-Poincare equations on Lie groups and homogeneous spaces, orbit invariants and applications, Lett. Math. Phys. 97 (2011), 45-60. 
[70] C. Vizman, Geodesic equations on diffeomorphism groups, SIGMA 4 (2008), no. 030, 22pp.

[71] W. Wolibner, Un theorème sur l'existence du mouvement plan d'un fluide parfait, homogene, incompressible, pendant un temps infiniment long, Math. Zeit. 37 (1933), 698-726.

[72] V. Yudovich, Non-stationary flows of an ideal incompressible fluid, Zhur. Vysch. Mat. Fiz. 3 (1963), no. 6, 1407-1456.

B. Khesin

Department of Mathematics, University of Toronto,

M5S 2E4, Canada

E-mail: khesin@math.toronto.edu

J. Lenells

Department of Mathematics, Baylor University,

One Bear Place \#97328, Waco, TX 76798, USA.

E-mail: Jonatan_Lenells@baylor.edu

G. Misiołek

Institute for Advanced Study,

Princeton, NJ 08540, USA

and

Department of Mathematics, University of Notre Dame, IN 46556, USA

E-mail: gmisiole@nd.edu

S. C. Preston

Department of Mathematics, University of Colorado, CO 80309, USA

E-mail: Stephen.Preston@colorado.edu 